\title{
Entomofauna do Oeste do Estado de Santa Catarina, Sul do Brasil
}

\author{
Mario Arthur Favretto ${ }^{\bowtie}$, Emili Bortolon dos Santos $^{2}$ \& Cleiton José Geuster²
}

1. Prefeitura Municipal de Campos Novos, Secretaria Municipal de Saúde, e-mail: marioarthur.favretto@hotmail.com (Autor para correspondência ${ }^{\varpi}$ ). 2. Universidade do Oeste de Santa Catarina, Campus de Joaçaba, e-mail: emilibortolon@hotmail.com, otabarana@yahoo.com.br.

\section{EntomoBrasilis 6 (1): 42-63 (2013)}

Resumo. No presente trabalho é apresentada uma lista de 1328 espécies de insetos observadas no oeste do Estado de Santa Catarina nas últimas oito décadas, sendo que esta riqueza de espécies equivale a 1,47\% do total de espécies registradas no Brasil. Foram compilados, em diversas coleções, os registros de exemplares coletados por F. Plaumann, foi consultada a literatura, e, além disso, foram feitas observações pessoais. Das 17 ordens registradas, Hymenoptera foi a que apresentou a maior riqueza de espécies, com 382, sendo que, a família Formicidae representou 48,42\% das espécies desta ordem e Apidae 19\%. Em Coleoptera foram registradas 230 espécies, com Curculionidae representando 22,60\% desse total e Coccinelidae 19,13\%. Na ordem Lepidoptera foram 199 espécies, a família Nymphalidae representou 37,18\% dessa riqueza e Saturniidae 22,11\%. total registrado para o Brasil, mas, trata-se de uma etapa importante para ampliar os conhecimentos sobre a distribuição geográfica destas espécies.

Palavras-chave: Distribuição geográfica; Insecta; Lista de espécies; Riqueza.

\section{Entomofauna from West of Santa Catarina State, South of Brazil}

Abstract. In this study is presented a list of 1328 insect species observed in west of Santa Catarina State, Brazil, in the last eight decades. The species richness founds corresponds $1.47 \%$ of the total of species registered in Brazil. The data set was compiled from collection records performed by F. Plaumann, in addition to the records from literature and personal observations. Here, we recorded a total of 17 orders of insects. The order Hymenoptera had greatest richness with 382 species. Within Hymenoptera, the family Formicidae represented $48.42 \%$ and Apidae, 19\%. In order Coleoptera, we found 230 species, with Curculionidae representing $22.60 \%$ of this total and Coccinelidae $19.13 \%$. On the order Lepidoptera we recorded 199 species, the Nymphalidae family represented 37.18\% of this richness and Saturniidae, 22.11\%. The species richness presented here is minimal when compared to the total recorded for Brazil, but it is an important step to improve the knowledge about the geographic distribution of these insects.

Keywords: Geographical distribution; Insecta; List of species; Richness.

(U) s insetos são considerados o grupo animal dominante no planeta Terra. Estão presentes em praticamente todos os ambientes e em riqueza de espécies superam todos os outros animais (TRIPLEHoRn \& Johnson 2011). Acredita-se que no mundo existam aproximadamente um milhão de espécies de insetos, com expectativas de haver ainda entre 2,5 milhões e 10 milhões de espécies a serem descritas (Grimaldi \& ENGEL 2005; Carvalho 2012).

No Brasil estima-se que existam cerca de 90.000 espécies descritas (RAFAEL et al. 2012). Entretanto, ainda não existem uma estimativa e uma compilação da riqueza de insetos para o Estado de Santa Catarina. Possivelmente a família de Hexapoda mais bem estudada em Santa Catarina seja Formicidae com o trabalho de Silva (1999), no qual são abordadas as espécies de formigas que ocorrem em todo o Estado. As demais ordens possuem apenas estudos regionais, porém não menos importantes. Dessa forma, o presente trabalho teve por objetivo reunir informações disponíveis na literatura, coleções entomológicas, além de observações pessoais dos autores sobre a ocorrência de insetos na região oeste do Estado de Santa Catarina, Brasil.

\section{MATERIAL E MÉTODOS}

O presente trabalho foi realizado entre novembro de 2011 e junho de 2012, período em que foi feita uma revisão sobre estudos de levantamento de espécies de insetos na região oeste de Santa Catarina (Figura 1). Esta região caracteriza-se pelo predomínio de floresta ombrófila mista, havendo também áreas com floresta estacional decidual em sua porção meridional e campos de altitude em sua porção setentrional próximo a divisa com o Paraná (KLEIN 1978).

Após foram verificados artigos de descrição de espécies e revisões taxonômicas que mencionassem exemplares coletados na região. Posteriormente foram realizadas buscas por informações em coleções científicas cujas informações sobre as espécies identificadas encontram-se disponíveis na literatura. Nas ordens Lepidoptera, Coleoptera e Hymenoptera também foram acrescentados dados de observações pessoais, em que as identificações basearam-se em comparações com o acervo do Museu Frei Miguel (Luzerna, SC), cuja coleção possui diversos exemplares doados por Julius Melzer e nas seguintes referências: Silva (1907), Costa-Lima (1945, 1950), Uehara-Prado et al. (2004).

Para a consulta em coleções científicas foram utilizados seus sistemas digitais, realizando buscas por insetos coletados por F. Plaumann ou por municípios da região oeste de Santa Catarina. As coleções das seguintes instituições foram consultadas: Coleção do Museu de Entomologia da FEIS/Unesp (MEFEIS), Coleção do Museu de Ciência e Tecnologia da PUCRS (MTC/ 


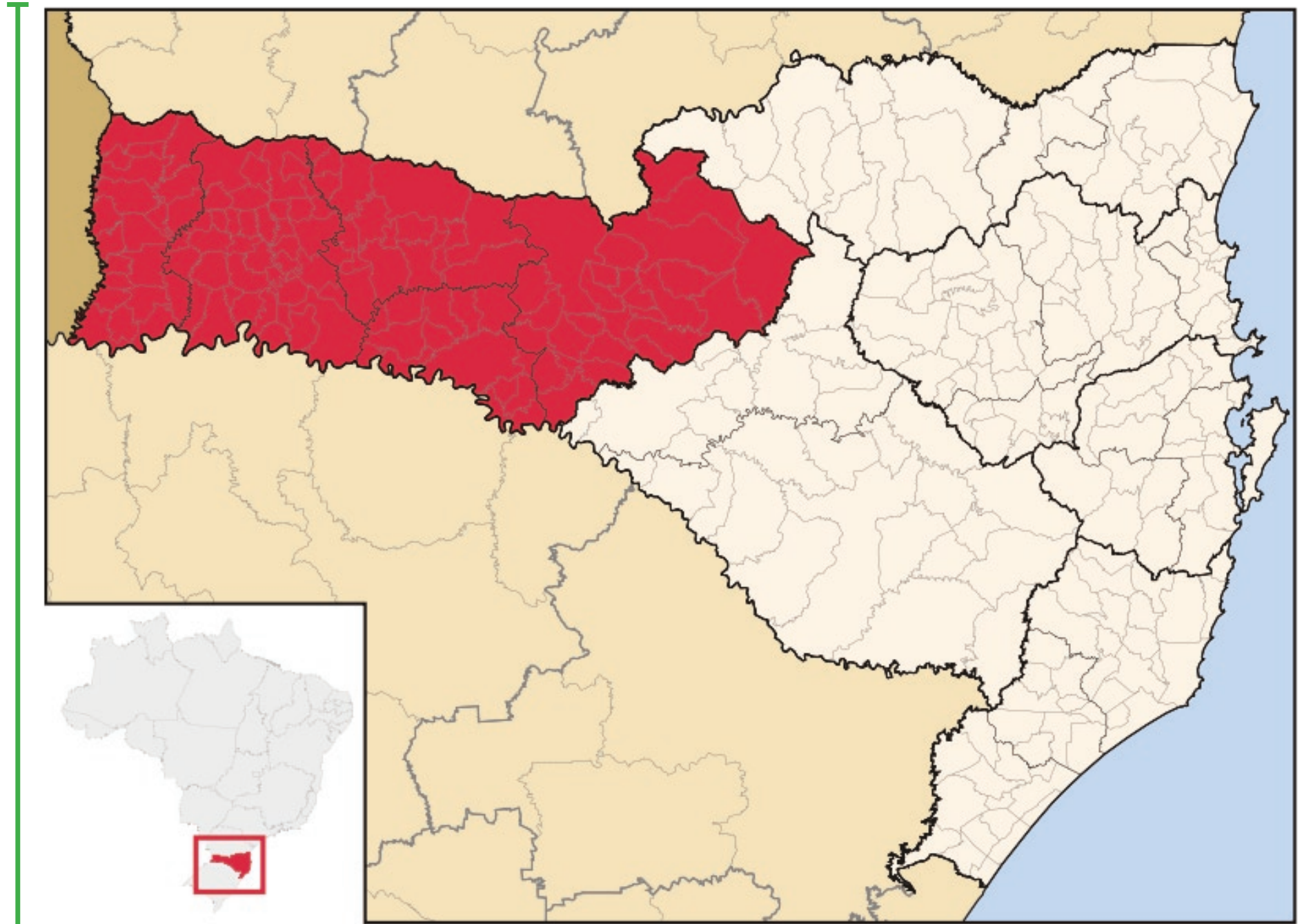

Figura 1. Em vermelho, localização da região oeste do Estado de Santa Catarina, Sul do Brasil.

Fonte: Wikipedia (http://pt.wikipedia.org/wiki/Ficheiro:SantaCatarina_Meso OesteCatarinense.svg).

PUCRS), Coleção Entomológica Pe. Jesus Santiago Moure (DZUP), Coleção Entomológica Paulo Nogueira Neto - IB/USP (CEPANN), Coleção Entomológica Adolph Hempel do Instituto Biológico (CEAH), Illinois Natural History Survey: Insect Collection (INHS), Yale Peabody Museum (YPM) e Smithsonian Institution (NMNH/SI),

As referências consultadas por meio de buscas no Google Scholar foram às seguintes: FLUKE (1937); BEQUART (1943); FLUKE (1945); Fisher (1947); ZiKAN \& WYGODZINSKY (1948); FisHER (1952); CAMRAS (1957); Guimarães (1961, 1963); Reichardt (1964); Evans (1965, 1966); Allen (1967); Martins (1971); Grazia-Vieira (1972); Meinander (1974); Hermann (1979); DeLong (1980a, 1980b); Johnson (1981); Gerdes (1984); Kimsey (1985); Coelho et al. (1989); Wirth \& Felippe-Bauer (1989); ShafFer (1991); Wirth (1991); Marinoni et al. (1992); Wirth \& SPINELli (1992); Bicho \& Bohart (1993); Urban (1993); Almeida \& Lima (1995); DomíngueZ (1995); GraF (1995); Juñente \& Loiacono (1995); Martins \& Galileo (1995); Urban (1995a, 1995b); Lugo-Ortiz \& Martins \& Galileo (1996); McCafferty (1996a, 1996b); Penteado-Dias (1996); Geller-Grimm (1997); Graf \& Fumagai (1997); Martins \& Galileo (1997); Milleo et al. (1997); Zaballos \& Mateus (1997); Almeida (1998); Dias (1998); NApp \& Reynaud (1998); Bremer \& Triplehorn (1999); Graf \& Marzagão (1999); Silva (1999); FonseCA \& Vieira (2000); HANSSON (2000); RothERAY et al. (2000); Galileo \& Martins (2001); Hickel et al. (2001); Loí́CONO et al. (2001); Martins \& Galileo (2001); Molineri (2001); TOMA (2001); Nieser \& Lopez-Ruf (2001); Toma \& Guimarães (2001); Borowiec (2002); Graf \& Fumagai (2002); Nunez \& Couri (2002); Nieser \& Chen (2002); Urban (2002); Garcia et al. (2003); Graciolli (2003); Gumovsky \& Boucek (2003); Irmler (2003); Stebnicka (2003); Toma (2003); Zanol (2003); Santos-Silva
\& Martins (2004); Trematerra \& Brown (2004); Ale-Rocha (2004); MusetTI \& JohnSon (2004); STEBNicka (2004); URURAhYRodrigues (2004); Fortes \& Grazia (2005); Galileo \& Martins (2005); Graciani et al. (2005); Stebnicka (2005); Araujo-Siqueira \& Almeida (2006); Marcondes et al. (2006); Nihei \& Pansonato (2006); Hovore \& Santos-Silva (2007); NAPP (2007); Ravanello (2007); Stebnicka (2007); Wendt \& CARVAlHo (2007); Caterino \& TishechKin (2008); Costa et al. (2008); Kerzhner \& Konstantinov (2008); Krug \& Alves-dos-Santos (2008); Lamas \& Mellinger (2008); Martins \& Galileo (2008); Scarbrough (2008); ShPEley \& Ball (2008); Galileo \& Martins (2009); Gomes et al. (2009); Irmler (2009); Napp (2009); Ribeiro \& Estévez (2009); WENDT \& Carvalho (2009); Ferreia \& Henry (2010); Aragão \& Monné (2011); Clarke et al. (2011); Froehlich (2011); Jesús-Bonilla et al. (2011); Lutinski et al. (2011); Moreira et al. (2011); SHIMBORI \& Penteado-Dias (2011); Favretto (2012).

\section{RESULTADOS E DISCUSSÃO}

Foram registradas 1328 espécies de insetos no oeste de Santa Catarina (Tabela 1). Ao todo foram registradas 17 ordens, sendo que as ordens com maior riqueza de espécies foram Hymenoptera (382 espécies), Coleoptera (230 espécies) e Lepidoptera (199 espécies) (Figura 2). Na Mata Atlântica do Estado do Rio de Janeiro, em um levantamento usando metodologia similar CouRI et al. (2009), registraram 3120 espécies de insetos, e as ordens com maior riqueza de espécies foram Coleoptera (1314 espécies), Diptera (659 espécies) e Hemiptera (453 espécies).

Adicionalmente, as famílias com maior riqueza foram Formicidae com 13,85\% ( $\mathrm{n}=184$ espécies) do total de espécies registradas, seguida das famílias Nymphalidae e Apidae, ambas com 5,57\% 
$(n=74)$ de espécies e Phlaeothripidae com 5,12\% ( $\mathrm{n}=68)$ (Figura 3), nenhuma destas famílias foi abordada por Couri et al. (2009). A ordem que apresentou maior número de famílias foi Hemiptera com 24 famílias, seguida de Hymenoptera com 21 e Coleoptera com 20 (Figura 4).

No estudo de Couri et al. (2009), a família que apresentou maior riqueza de espécies foi Cerambycidae com 1149 representantes, enquanto no presente trabalho foram registradas apenas 43 espécies para esta família. A segunda família que apresentou maior riqueza para Couri et al. (2009) foi Cicadellidae com 340 espécies, que, no presente estudo foi representada por 40 espécies.

Dentre as famílias com maior riqueza de espécies no presente estudo, Formicidae (Hymenoptera) representa 7,36\% do total de espécies conhecidas desta família para o Brasil, Apidae representou 4,41\% do total registrado no Brasil (Melo et al. 2012), Nymphalidae representou 9,25\% da riqueza de espécies nacional (DUARTE et al. 2012), Phlaeothripidae (Thysanoptera) representou 19,26\% das espécies com ocorrência registrada para o Brasil (MONTEIRo \& MOUND 2012).

No oeste de Santa Catarina existem alguns levantamentos realizados com algumas ordens de Hexapoda que ajudam expor um pouco da diversidade desta classe na região em questão. Com a ordem Odonata, há o trabalho de Ravanello (2007), em que foi realizado um levantamento em nove rios da região (rios Irani, Xaxim, Taquaruçu, Lajeado Bonito, Lambedor, Lajeado São José, Iracema, Palmitos e São Domingos). Com a ordem Orthoptera existem os estudos de Graciani et al. (2005) e Lutinski et al. (2011), ambos realizados no município de Chapecó.
Na ordem Diptera há o trabalho de MARCONDEs et al. (2006) sobre Culicidae e o trabalho de GARCIA et al. (2003) sobre Tephritidae. Para a ordem Hymenoptera há o trabalho de KRUG \& ALVESDOS-SANTOS (2008) em Porto União com as famílias Halictidae, Apidae, Megachilidae, Andrenidae e Colletidae. Enquanto que com a família Formicidae podem ser citados os trabalhos de Silva (1999) abrangendo todo o oeste de Santa Catarina, Silva \& Silvestre (2000, 2004) no município de Seara, Lutinski \& Garcia (2005) e LuTinski et al. (2008) no município de Chapecó.

Relacionado a ordem Lepidoptera há o estudo de FAVRETto (2012) no município de Joaçaba. Referente à ordem Hemiptera há o trabalho de Hickel et al. (2001) em Videira e com insetos de uma forma geral há o trabalho de GARCiA et al. (2004) em lavouras de milho (Zea mays Linnaeus) no município de Arvoredo.

Percebe-se que são poucos os trabalhos sistematizados que se dedicam ao levantamento de insetos na região oeste de Santa Catarina, tornando sua entomofauna ainda pouco estudada. Esta ausência de dados pode implicar em decisões erradas quanto a ações conservacionistas na região, havendo então a importância de se conhecer esta parcela da fauna.

Desta forma, no presente trabalho é apresentada uma lista que representa uma pequena parcela da biodiversidade da região oeste de Santa Catarina, pois 1328 espécies é um valor baixo, representando $1,47 \%$ de uma riqueza de espécies estimada em 90000 para o Brasil (RAFAEL et al. 2012). No entanto, certamente esse é o primeiro passo na reunião de informações desta natureza, permitindo o rápido acesso ao conhecimento agora disponível neste trabalho.

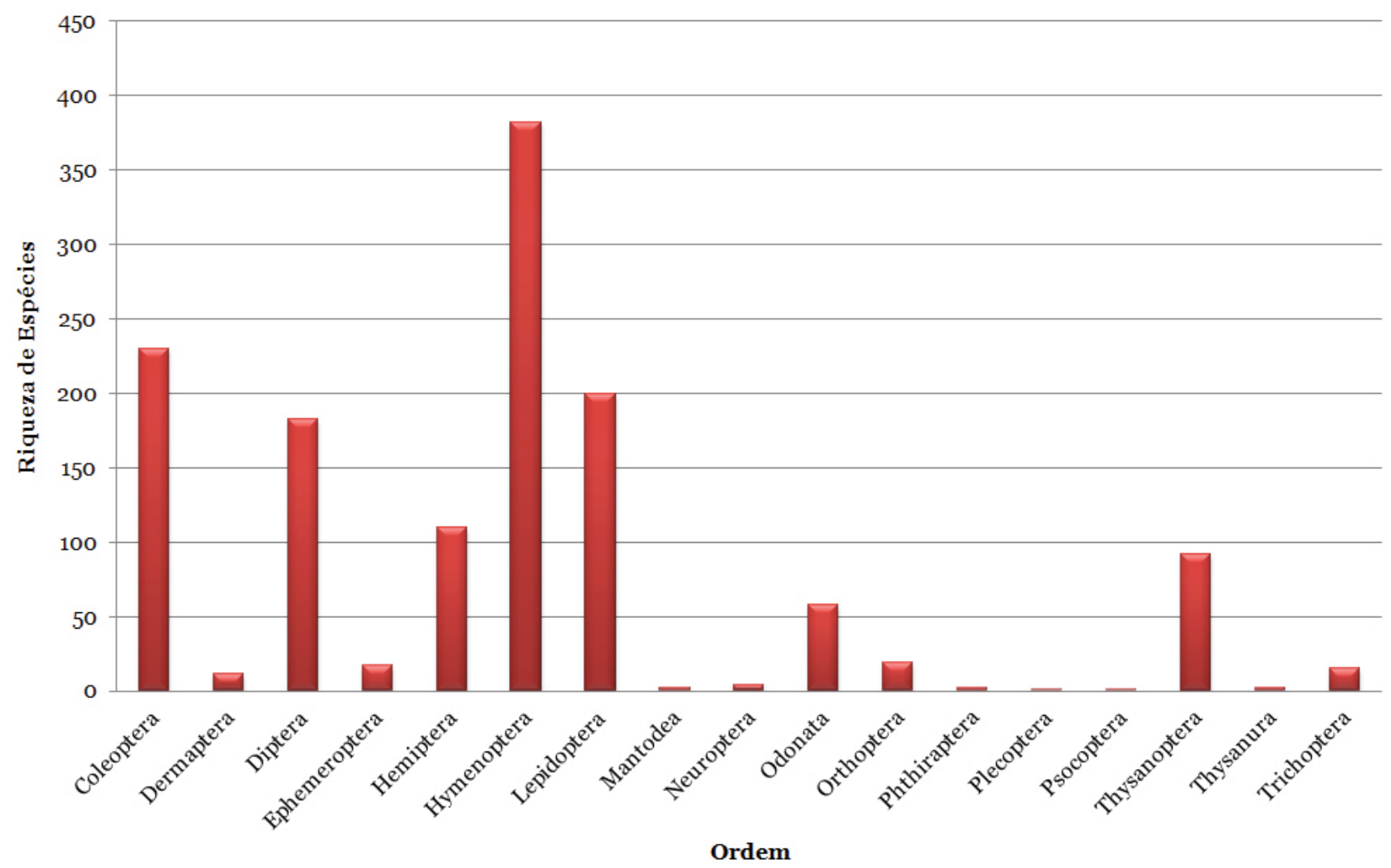

Figura 2. Riqueza de espécies por ordem de Hexapoda registradas no oeste do Estado de Santa Catarina, Brasil. 


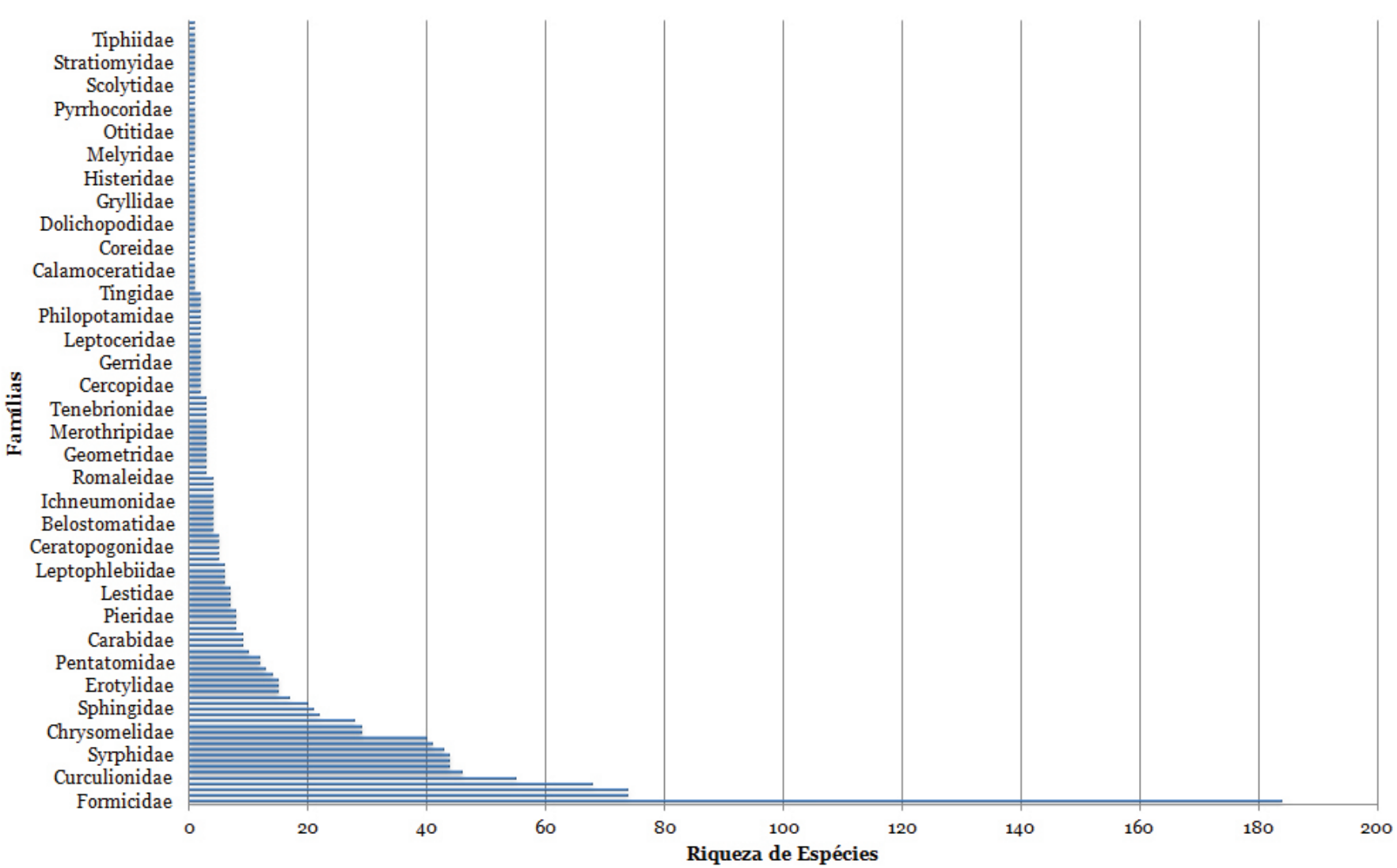

Figura 3. Riqueza de espécies em cada família registradas no oeste do Estado de Santa Catarina, Brasil.

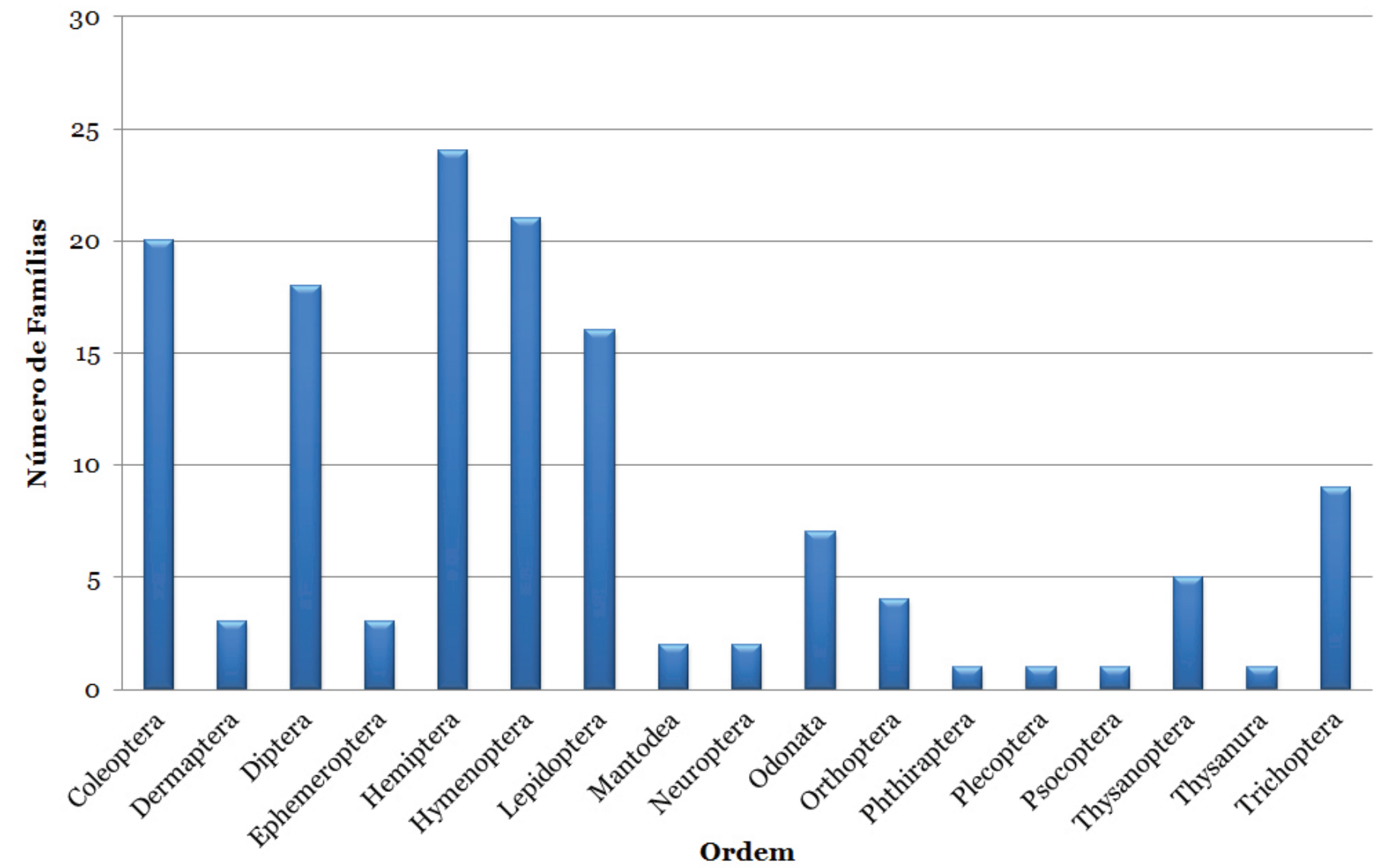

Figura 4. Número de famílias registradas em cada ordem no oeste do Estado de Santa Catarina, Brasil. 
Tabela 1. Lista das espécies de insetos registradas no oeste do Estado de Santa Catarina, Brasil.

\begin{tabular}{|c|c|}
\hline Ordem/ Família & Espécie \\
\hline & Thysanura \\
\hline \multirow{2}{*}{ Machilidae } & Machilellus plaumanni Wygodzinsky \\
\hline & Machilellus santacatarinensis Wygodzinsky \\
\hline \multicolumn{2}{|r|}{ Plecoptera } \\
\hline Perlidae & Kempnyia kaingang Froehlich \\
\hline \multicolumn{2}{|r|}{ Mantodea } \\
\hline Vatidae & Brunneria longa Giglio-Tos \\
\hline Mantoididae & Mantoidea luteola Westwood \\
\hline \multicolumn{2}{|r|}{ Psocoptera } \\
\hline Psocidae & Psococerastis fasciata Mockford \\
\hline \multicolumn{2}{|r|}{ Phthiraptera } \\
\hline \multirow{2}{*}{ Hoplopleuridae } & Hoplopleura fonsecai Werneck \\
\hline & Hoplopleura affinis Burmeister \\
\hline \multicolumn{2}{|r|}{ Ephemeroptera } \\
\hline \multirow{5}{*}{ Baetidae } & Americabaetis alphus Lugo-Ortiz \& McCafferty \\
\hline & Americabaetis labiosus Lugo-Ortiz \& McCafferty \\
\hline & Americabaetis titthion Lugo-Ortiz \& McCafferty \\
\hline & Paracloeodes leptobranchus Lugo-Ortiz \& McCafferty \\
\hline & Cryptonympha copiosa Lugo-Ortiz \& McCafferty \\
\hline \multirow{4}{*}{ Leptohyphidae } & Tricorythopsis gibbus Allen \\
\hline & Leptohyphes plaumanni Allen \\
\hline & Trichorythodes barbus Allen \\
\hline & Trichorythodes bullus Allen \\
\hline \multirow{6}{*}{ Leptophlebiidae } & Thraulodes traverae Thew \\
\hline & Thraulodes daidaleus Thew \\
\hline & Ulmeritoides uruguayensis Traver \\
\hline & Ulmeritoides patagiatus Thew \\
\hline & Ulmeritus balteatus Thew \\
\hline & Ulmeritoides haarupi Esben-Petersen \\
\hline & Odonata \\
\hline
\end{tabular}

\section{Anisoptera}

\begin{tabular}{l|l|}
\hline & Castoraeschna sp. \\
\hline & Coryphaeschna sp. \\
\hline Gynacantha sp. \\
\hline Limnetron sp. \\
\hline Rhionaeschna sp. \\
\hline Remartinia sp. \\
\hline Aeshna sp. \\
\hline Rhionaeschna cornigera Brauer \\
\hline Phyllocycla sp. \\
\hline Progomphus sp. \\
\hline Tibiogomphus sp. \\
\hline Gomphoides sp. \\
\hline Aphylla sp. \\
\hline Orthemis plaumanni Buckhholz \\
\hline Brechmorhoga sp. \\
\hline Cannaphila sp.
\end{tabular}

Libellulidae

\section{Ordem/ Família}

Espécie

Dythemis sp.

Elasmothemis sp.

Erythrodiplax sp.

Erythrodiplax connata Burmeister

Gynothemis sp.

Idiataphe sp.

Macrothemis sp.

Micrathyria sp.

Neocordulia sp.

Orthemis sp.

Pantala sp.

Pantala flavescens Fabricius

Perithemis sp.

Perithemis mooma Kirby

Perithemis waltheri Ris

Planiplax sp.

Tramea sp.

Tramea cophysa Hagen

Zygoptera

\begin{tabular}{ll}
\hline Calopterygidae & Hetaerina $\mathrm{sp}$. \\
\cline { 2 - 2 } & Mnesarete $\mathrm{sp}$.
\end{tabular}

Argentagrion ambiguum Ris

Homeoura chelifera Selys

Acanthagrion sp.

Argia sp.

Argia amethystoides Cook

Ischnura sp.

Coenagrionidae

Ischnura capreolus Hagen

Ischnura fluviatilis Selys

Leptobasis sp.

Oxyagrion sp.

Oxyagrion terminale Selys

Telebasis carmesina Calvert

Telebasis theodori Navás

Megapodagrionidae Heteragrion sp.

Lestes auritus Hagen in Selys

Lestes bipupullatus Calvert

Lestes paulistus Calvert

Lestidae

Lestes pictus Hagen in Selys

Lestes quadristriatus Calvert

Lestes spatula Fraser

Lestes tricolor Erichson

\section{Orthoptera}

Abracris flavolineata De Geer

Allotruxalis strigata Giglio-Tos

Amblytropidia sola Rehn

Acrididae

Cylindrotettix chacoensis Roberts

Dichroplus misionensis Carbonell

Eurotettix lilloanus Liebermann 


\begin{tabular}{|c|c|}
\hline Ordem/ Família & Espécie \\
\hline \multirow{8}{*}{ Acrididae } & Metaleptea brevicornis adspersa Johannson \\
\hline & Orphulella punctata De Geer \\
\hline & Parorphula graminea Bruner \\
\hline & Rhammatocerus brunneri Giglio-Tos \\
\hline & Ronderosia bergi Stal \\
\hline & Schistocerca flavofasciata De Geer \\
\hline & Cornops aquaticum Bruner \\
\hline & Cornops frenatum Roberts \& Carbonell \\
\hline \multirow{4}{*}{ Romaleidae } & Chromacris speciosa Thunberg \\
\hline & Staleochlora viridicata Serville \\
\hline & Xyleus discoideus Serville \\
\hline & Zoniopoda tarsata Serville \\
\hline \multicolumn{2}{|l|}{ Tridactylidae } \\
\hline Gryllidae & Gryllus assimilis Fabricius \\
\hline \multicolumn{2}{|r|}{ Dermaptera } \\
\hline \multirow{2}{*}{ Forficulidae } & Doru sp. \\
\hline & Doru luteipes Scudder \\
\hline \multirow{2}{*}{ Labiduridae } & Labidura xanthopus Stal \\
\hline & Labidura riparia Pallas \\
\hline \multirow{7}{*}{ Spongiphoridae } & Spongiphora bormansi Burr \\
\hline & Spongiphora buprestoides Kirby \\
\hline & Spongiphora croceipennis Serville \\
\hline & Vostox sp. \\
\hline & Sparatta sp. \\
\hline & Kleter sp. \\
\hline & Ancistrogaster sp. \\
\hline
\end{tabular}

\section{Ordem/ Família}

Hoplothrips testaceus Hood

Lissothrips eburifer Hood

Terthrothrips viduus Hood

Terthrothrips bollifer Hood

Lissothrips ventralis Hood

Terthrothrips irretitus Hood

Copiothrips brasiliensis Hood

Lissothrips flavidus Hood

Lissothrips tricosus Hood

Eurythrips alarius Hood

Malacothrips fasciatus Hood

Eurythrips hemimeres Hood

Schazothrips anadenus Hood

Erksothrips bucca Hood

Chthonothrips nigrocinctus Hood

Eurythrips umbrisetis Hood

Terthrothrips hebes Hood

Copiothrips ustulatus Hood

Eurythrips elongatus Hood

Eurythrips conformis Hood

Eurythrips musivi Hood

Trypanothrips coxalis Hood

Terthrothrips bucculentus Hood

Phlaeothripidae

Eurythrips striolatus Hood

Chorithrips heptatoma Hood

Chamaeothrips jucundus Hood

Malacothrips tesselatus Hood

Gastrothrips fumipennis Hood

Terthrothrips balteatus Hood

Symphyothrips caliginosus Hood

Phthirothrips longiceps Hood

Eurythrips nigriceps Hood

Mystrothrips clavatoris Hood

Eurythrips occipitalis Hood

Eurythrips cruralis Hood

Preeriella marginata Hood

Terthrothrips carens Hood

Eschatothrips cerinus Hood

Preeriella fumosa Hood

Terthrothrips peltatus Hood

Porcothrips citricornis Hood

Terthrothrips irretitus Hood

Terthrothrips serratus Hood

Eurythrips peccans Hood

Carathrips impensus Hood

Holothrips amplus Hood

Preeriella discors Hood 


\section{Ordem/ Família}

Chirothrips nigriceps Hood

Frankliniella desantisi Sakimura \& O’Neill

Frankliniella konoi Sakimura \& O’Neill

Leucothrips pictus Hood

Plesiothrips maculosus Hood

Prionothrips procerus Hood

Thripidae

Pseudodendrothrips alboniger Hood

Pseudothrips interruptus Hood

Schistothrips quadratus Hood

Sericothrips daedalus Hood

Sericothrips fimbriatus Hood

Sericothrips hemileucus Hood

Franklinothrips lineatus Hood

Aeolothripidae

Stomatothrips rotundus Hood

Erythrothrips loripes Hood

Heterothrips decoratus Hood

Heterothrips angusticeps Hood

Heterothripidae

Aulacothrips dictyotus Hood

Heterothrips bicolor Hood

Merothrips tympanis Hood

Merothripidae

Merothrips mirus Crawford

Merothrips plaumanni Crawford

$\frac{\text { Hemiptera }}{\text { Belostoma candidulum Montandon }}$

Belostomatidae

Belostoma sanctulum Montandon

Belostoma noualhieri Montandon

Belostoma plebejum Sta

Limnogonus ignotus Drake \& Harris

Gerridae

Metrobates vigilis Drake

Helotrephidae

Neotrephes usingeri China

Mayrinia curvidens Mayr

Serdia concolor Ruckes

Serdia indistincta Fortes \& Grazia

Serdia limbatipennis Stal

Serdia bicolor Fortes \& Grazia

Serdia maxima Fortes \& Grazia

Pentatomidae

Serdia calligera Stal

Nezara viridula Linnaeus

Piezodorus guildinii Westwood

Edessa sp.

Podisus nigrispinus Dallas

Acrosternum brasicola Rolston

Nabidae

Pagasa fuscipennis Reuter

Ambracius dufouri Stal

Fulvius guapimirinus Carvalho \& Costa

Miridae

Fulvius quadristillatu Carvalho \& Costa

Fulvius stillatipennis Stal

\begin{tabular}{|c|c|}
\hline Ordem/ Família & Espécie \\
\hline \multirow{3}{*}{ Naucoridae } & Limnocoris aculabrum La Rivers \\
\hline & Limnocoris maculiceps Montandon \\
\hline & Limnocoris pusillus Montandon \\
\hline \multirow{2}{*}{ Tingidae } & Corythucha clara Drake \& Hambleton \\
\hline & Zetekella pulla Drake \& Plaumann \\
\hline \multirow{6}{*}{ Reduvidae } & Pselliopus sp. \\
\hline & Stenolemus plaumanni Wygodzinsky \\
\hline & Zelurus brunneus plaumanni Lent \& Wygodzinsky \\
\hline & Zelus sp. \\
\hline & Elasmocoris comptus Harris \& Drake \\
\hline & Reduvis armillatus Lethierry \& Severin \\
\hline Pyrrhocoridae & Geocoris sp. \\
\hline Delphacidae & Columbisoga (Columbisodes) saracura Fennah \\
\hline Aetalionidae & Aethalion reticulatum Linnaeus \\
\hline \multirow{2}{*}{ Cercopidae } & Deois schach Fabricius \\
\hline & Deois flavopicta Stall \\
\hline \multirow{31}{*}{ Cicadellidae } & Subrasaca austera Young \\
\hline & Molomea lineiceps Young \\
\hline & Sonesimia grossa Signoret \\
\hline & Balclutha obunca Blocker \\
\hline & Subrasaca atronasa Young \\
\hline & Nullana elbana Delong \& Martinson \\
\hline & Agallia albidula Uhler \\
\hline & Agalliana ensigera Oman \\
\hline & Bucephalogonia xenthophis Berg \\
\hline & Dilobopterus díspar Germar \\
\hline & Ferrariana trivittata Signoret \\
\hline & Hortensia similis Walker \\
\hline & Oncometopia sp. \\
\hline & Plesiommata corniculata Young \\
\hline & Syncharina punctatissima Signoret \\
\hline & Amblicephalus marginellanus faminoides Linnavuori \\
\hline & Atanus sp. \\
\hline & Bahita sp. \\
\hline & Balcluta hebe Kerk \\
\hline & Chlorotettix minimus Baker \\
\hline & Copididonus vittulatus Berg \\
\hline & Exitianus obscurinervis Stal \\
\hline & Graminella striatella Linnavuori \\
\hline & Haldorus sp. \\
\hline & Menosoma cincta Osborn \& Ball \\
\hline & Osbornellus lamellaris Linnavuori \\
\hline & Planicephalus flavitta Berg \\
\hline & Spangbergiella uruguayensis Berg \\
\hline & Stirellus picinus elegantulus Linnavuori \\
\hline & Unerus colonus Uhler \\
\hline & Frequenamia cavifrons Linnavuori \\
\hline
\end{tabular}

mocoris maculiceps Montandon

Limnocoris pusillus Montandon

clara Drake \& Hambleton

ella pulla Drake \& Plaumann

Pselliopus $\mathrm{sp}$

olemus plaumanni Wygodzinsky

Zelurus brunneus plaumanni Lent \& Wygodzinsky

Elasmocoris comptus Harris \& Drake

mbisoga (Columbisodes) saracura Fennah

Aethalion reticulatum Linnaeus

ois schach Fabricius

Deois flavopicta Stall

lineiceps Young

Agllia albidula Uhler

Dilobopterus díspar Germa

tensia similis Walker

mata corniculata Young

Syncharina punctatissima Signoret

marginellanus faminoides Linnavuori

tanus sp.

Chlorotettix minimus Baker

Haldorus sp.

Menosoma cincta Osborn \& Bal

Osbornellus lamellaris Linnavuor

Planicephalus flavitta Berg

Spangbergiella uruguayensis Berg

inus elegantulus Linnavuori

Continua.. 


Trdem/ Família $\quad$ Espécie

Frequenamia saranensis Osborn

Frequenamia spiniventris Linnavuori

Gypona sp.

Cicadellidae

Gypona vireta DeLong

Gypona anfracta Delong \& Linnavori

Gypona lasua DeLong

Empoasca sp.

Protalebrella brasiliensis Baker

Ceresa ustulata Fairmaire

Membracidae Ceresa brunnicornis Germar

Philya inflata Metcalf

\begin{tabular}{ll} 
Aphididae & Rhopalosiphum $\mathrm{sp}$. \\
\hline Cicadidae & Cicada sp. \\
\hline & Ischnodemus proprius Slater
\end{tabular}

Lygaeidae

Cymus sp.

Xenoblissus lutzi Barber

Dallasiellus ovalis Froeschner

Cydnidae

Onalips neogeus Froeschner

Glyptocoris sejunctus Harris \& Drake

Pictinus fictus Kormilev

Pictinus rhombocarinata Kormilev

Mezira fritzi Kormilev

Notapictinus terminalis Kormilev

Dihybogaster plana Kormilev

Neuroctenus longuiusculus Kormilev

Aradidae

Dihybogaster plaumanni Kormilev

Glyptocoris confusus Kormilev

Diphyllonotus brachypterus Kormilev

Aneurus plaumanni Kormilev

Artagera plaumanni Kormilev

Calisius brasiliensis Kormilev

Calisiopsis brasiliensis Kormilev

Pictinus brachypterus Drake \& Kormilev

Microvelia novana Drake \& Plaumann

Veliidae

Microvelia arca Drake

Husseyella halophila Drake

Coreidae Leptoglossus lonchoides Allen

Enicocephalidae Oncylocotes sp.

Saldidae Saldula scitula Drake \& Hottes

Coleoptera

Trachyderes thoracicus Oliver

Acanthoderes versicolor Melzer

Ischasia cuneiformis Fisher

Ischasia cazieri Fisher

Cerambycidae

Odontocera bivitticollis Fisher

Odontocera melzeri Fisher

Ommata (Eclipta) lateralis Fisher

Ommata (Eclipta) brasiliensis Fisher

\section{Ordem/ Família}

Espécie

Ommata (Rhopalessa) atripes Fisher

Rhopalessa clavicornis Bates

Rhopalessa demissa Melzer

Estolomimus solidus (=Neostola solida?) Breuning

Estolomimus curtus (=Estola curta) Breuning

Estolomimus pulvereus Martins \& Galileo

Xenofrea apicalis Melzer

Xenofrea albofasciata Galileo \& Martins

Anisopeplus perplexus Melzer

Rhopalophora neivai Mendes

Rhopalophora collaris Germar

Cometes hirticornis Lepeletier \& Audinet-Serville

Aleiphaquilon plaumanni Martins

Gnomidolon v. varians Gounelle

Tetraibidion ephimerum Martins

Minibidion minusculum Martins

Tropidion s. signatum Serville

Cerambycidae

Compsibidion sp.

Compsa multiguttata Melzer

Urangaua analis Melzer

Megacyllene (Megacyllene) unicolor Fuchs

Megacyllene (Megacyllene) mellyi Chevrolat

Megacyllene (Megacyllene) castanea Laporte \& Gory

Hesycha inermicollis Breuning

Aglaoschema viridipenne Thomson

Aglaoschema erythrocephala Thomson

Aglaoschema ventrale Germar

Aglaoschema prasinipenne Lucas

Cometes melzeri Santos-Silva \& Martins

Callisema socium Martins \& Galileo

Oncideres captiosa Martins

Callideriphus flavipennis Melzer

Coccoderus novempunctatus Germar

Psygmatocerus wagleri Perty

Otoglossa tuberculosa Chaudoir

Tetragonoderus deuvei Shpeley \& Ball

Tetragonoderus quadriguttatus Dejean

Helluobrochus ares Reichardt

Carabidae

Lebia tendicula Liebke

Lebia concina Germar

Cicindela sp. Linnaeus

Anillinus minor Zaballos \& Mateu

Anillinus magnus Zaballos \& Mateu

Agroiconota inedita Boheman

Agroiconota tristriata Fabricius

Charidotella immaculata Olivier

Charidotella recidiva Spaeth

Continua.. 


\begin{tabular}{|l|l|}
\hline Ordem/ Família & \multicolumn{1}{c}{ Espécie } \\
\hline Charidotella vinula Boheman \\
\hline Charidotis auroguttata Boheman \\
\hline Charidotis consentanea Boheman \\
\hline Charidotis gemellata Boheman \\
\hline Charidotis mansueta Boheman \\
\hline Charidotis ocularis Boheman \\
\hline Chelymorpha inflata Boheman \\
\hline Coptocycla adamantina Boheman \\
\hline Coptocycla fastidiosa Boheman \\
\hline Cteisella confusa Boheman \\
\hline Cteisella ramosa Spaeth \\
\hline Hybosa acutangula Spaeth
\end{tabular}

Chrysomelidae

\section{Maecolaspis joliveti Bechyne}

Metriona elatior Klug

Microctenochira optata Boheman

Microctenochira similata Boheman

Plagiometriona punctatissima Boheman

Plagiometriona tenella Klug

Cephaloleia linkei Uhmann

Hibosispa nítida Uhmann

Xenochalepus trilineatus utraque Uhmann

Diabrotica speciosa Germar

Cephalobia sp.

Clinorispia sp.

Pavadicatelia sp.

Gordonoryssomus deyrollei Crotch

Gordonoryssomus delicatus Almeida \& de Moura

Lima

Neocalvia anastomozans Crotch

Neocalvia fulgurata Mulsant

Exoplectra columba Costa, Almeida \& Correa

Cycloneda sanguinea Linnaeus

Cycloneda pulchella Klug

Hinda uncinata Mulsant

Cyra ceciliae Crotch

Cyra hibridula Crotch

Cyra glyphica Mulsanzt

Psyllobora camargoi Almeida

Psyllobora plaumanni Almeida

Achryson surinamum Linnaeus

Aulis guttata Sicard

Azia bioculata Gordon

Brachiantha sp.

Coleomegilla quadrifasciata Schönherr

Cryptognatha sp.

Eriopis conexa Germar

Hyperaspis sp. 1

Hyperaspis sp. 2

\section{Ordem/ Família}

Espécie

Hyperaspis sp. 3

Hyperaspis sp. 4

Hyperaspis ceciliae Crotch

Hyperaspis crucifera Mulsant

Hyperaspis hexastigma Mulsant

Hyperaspis matronata Mulsant

Hyperaspis quadrina Mulsant

Neda callispilota Guérin-Méneville

Oryssomus subterminatus Mulsant

Pentilia egena Mulsant

Pseudoazya nana Marshall

Coccinellidae

Pseudoryssomus sp.

Psyllobora bicongregata Boheman

Psyllobora distinguenda Crotch

Psyllobora hybrida Mulsant

Psyllobora gratiosa Mader

Psyllobora meticulosa Mulsant

Psyllobora nana Mulsant

Rhizobius lophantae Blaisdell

Scymnus sp.

Stethorus sp.

Zagloba sp.

Histeridae

Lymexylidae

Hippeustister sp.

Melitomma brasiliense Murray

Melitomma sp.

Ataenius clitellarius Petrovitz

Ataenius lenkoi Petrovitz

Ataenius plaumanni Petrovitz

Ataenius londrinae Stebnicka

Ataenius catarinaensis Stebnicka

Scarabaeidae

Ataenius columbicus Harold

Canthon sp.

Enema cf. pan Fabricius

Gymnetis sp.

Megasoma hector Gory

Stereocephalus seriatipennis Arribalzaga

Staphylinidae

Nacaeus spegazzini Bernhauer

Corticeus notialis Bremer \& Triplehorn

Tenebrionidae

Corticeus abditus Bremer \& Triplehorn

Lagria villosa Fabricius

Hadromeropsis (Hadromeropsis) plebeia Howden

Omolabus plaumanni Voss

Pandeletrius sp.

Curculionidae

Steriobaris sp.

Themeropis sp.

Zygops sp.

Eucalandra luteosignata Blanchard

Continua... 


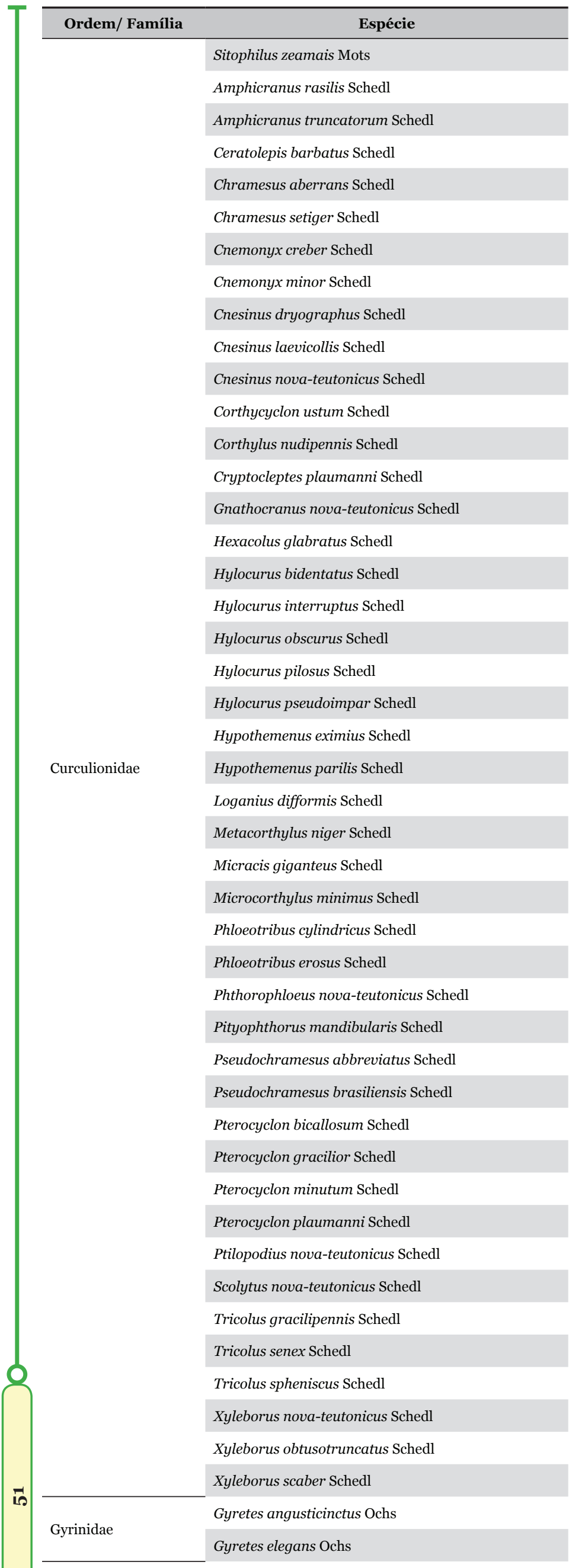

Ordem/ Família $\quad$ Espécie

\begin{tabular}{|c|c|}
\hline \multirow{4}{*}{ Gyrinidae } & Gyretes gibbosus Ochs \\
\hline & Gyretes latipes Ochs \\
\hline & Gyretes minusculus Ochs \\
\hline & Gyretes tarsalis Ochs \\
\hline Dytiscidae & Amarodytes plaumanni Gschwendtner \\
\hline Melyridae & Astylus variegatus Germar \\
\hline Hydraenidae & Parhydraenida sp. \\
\hline Colydiidae & Colydium catarinae Slipinski \\
\hline Corylophidae & Haplicnema sp. \\
\hline \multirow{3}{*}{ Cucujidae } & Scalidia cylindricollis Lacordaire \\
\hline & Catagenus asper Slipinski \\
\hline & Telephanus plaumanni Nevermann \\
\hline \multirow{10}{*}{ Erotylidae } & Aegithus chalybaeus Duponchel \\
\hline & Aegithus clavicornis Linnaeus \\
\hline & Aegithus sp. \\
\hline & Brachysphoe sp. 1 \\
\hline & Brachysphoe sp. 2 \\
\hline & Brachysphoe sp. 3 \\
\hline & Cypherotylus sp. \\
\hline & Gibbifer sp. \\
\hline & Iphiclus sp. \\
\hline & Poecilesthus sp. \\
\hline
\end{tabular}

Poecilesthus sp.

Octomaculatus sp.

Scaphidomorphus sp.

Mycotretus sp. 1

Mycotretus sp. 2

Mycotretus tigrinus Olivier

\begin{tabular}{l|l}
\hline Scolytidae & Pagiocerus punctatus Eggers \\
\hline & Elsianus aeolus Hinton \\
& Elsianus aequalis Hinton \\
& Elsianus avistatus Hinton \\
Elmidae & Elsianus celsus Hinton \\
& Elsianus isus Hinton \\
& Elsianus amanus Hinton \\
\hline Coniopterygidae & Coniopteryx (Scotoconiopteryx) tucumana Navs \\
\hline Coniopteryx (Coniopteryx) callangana Enderlein \\
\hline Hemerobiidae & Semidelis sp. \\
\hline & Hemerobius bolivari Banks \\
\hline
\end{tabular}

\section{Diptera}

Aedes crinifer Theobald

Aedes fluviatilis Lutz

Aedes hastatus/oligopistus Dyar

Aedes scapularis Rondani

Culicidae

Aedes serratus Theobald

Anopheles lutzi Cruz

Anopheles parvus Chagas

Anopheles strodei Root 


\section{Ordem/ Família}

Anopheles albitarsis Lynch Arribalzaga

Anopheles evansae Brethes

Anopheles galvaoi Causey, Deane \& Deane

Anopheles intermedius Peryassu

Anopheles parvus Chagas

Chagasia fajardi Lutz

Coquillettidia chrysonotum /albifera Prado

Coquillettidia juxtamansonia Chagas

Coquillettidia venezuelensis Theobald

Culex (Culex) dolosus/eduardoi Lynch Arribalzaga

Culex (Culex) gr. coronator Dyar \& Knab

Culex (Melanoconion) seção Melanoconion Theobald

Culex bigoti Bellardi

Culex quinquefasciatus Say

Limatus durhamii Theobald

Lutzia sp.

Culicidae

Streblidae

Mansonia wilsoni Barreto \& Coutinho

Ochlerotatus serratus complex Theobald

Ochlerotatus fluviatilis Lutz

Psorophora discrucians Walker

Psorophora lanei Shannon \& Cerqueira

Psorophora ciliata Fabricius

Psorophora ferox Humboldt

Runchomyia reversa Lane \& Cerqueira

Sabethes belisarioi Neiva

Sabethes identicus Dyar \& Knab

Sabethes melanonymphe Dyar

Sabethes albiprivus Theobald

Sabethes aurescens Lutz

Sabethes purpureus Theobald

Toxorhynchites sp.

Trichoprosopon pallidiventer Lutz

Wyeomyia limai Lane \& Cerqueira

Fannia obscurinervis Stein

Fannia penicillaris Stein

Fannia trimaculata Stein

Fannia tumidifemur Stein

Anatrichobius passosi Gracioli

Trichopsomyia currani Fluke

Trichopsomyia lasiotibialis Fluke

Trichopsomyia granditibialis Fluke

Epistrophe biarcuata Fluke

Xanthandrus plaumanni Fluke

Rhysops currani Fluke

Rhysops longicornis Williston

Rhysops fastigata Fluke

Rhysops nigrans Fluke

\section{Ordem/ Família}

Espécie

Rhysops columella Fluke

Rhysops lanei Fluke

Rhysops minuscula Fluke

Melanostoma lineata Fluke

Habromyia chrysotaenia Fluke

Ocyptamus luctuosus Bigot

Eristalis (Eristalis) tenax Linnaeus

Eristalis vera Hull

Eristalis cora Hull

Eristalis claripennis Hull

Allograpta hermosa Hull

Argentinomyia lineatus Fluke

Argentinomyia neotropicus Curran

Copestylum belinda Hull

Copestylum circumdatum Walker

Copestylum obscurior Curran

Copestylum pallens Wiedemann

Syrphidae

Copestylum spinithorax Lynch Arribalzaga

Copestylum tripunctatum Hull

Meromacrus niger Sack

Meromacrus pratorum Fabricius

Neplas sp.

Ocyptamus antiphates Walker

Ocyptamus calla Curran

Ocyptamus funebris Macquart

Palpada furcata Wiedemann

Palpada precipua Williston

Palpada rufoscutellata Sack

Paramicrodon flukei Curran

Pseudodoros sp.

Quinchuana sp.

Salpingogaster halcyon Hull

Sterphus shannoni Thompson

Syrphus phaeostigma Wiedemann

Xanthandrus nitidulus Fluke

Winthemia angusta Coelho, Carvalho \& Guimarães

Winthemia authentica Coelho, Carvalho \& Guimarães

Moreiria wiedemanni Toma \& Guimarães

Thysanopsis albicaudata Townsend

Adejeania bicaudata Curran

Avibrissosturmia nigra Guimaraes

Tachinidae

Avibrissosturmia plaumanni Guimaraes

Borgmeiermyia rozeni Arnaud

Chrysotachina viridis Nunez, Couri \& Guimaraes

Chrysotachina braueri Townsend

Chrysotachina panamensis Curran

Chrysotachina willistoni Curran 


Ordem/ Família $\quad$ Espécie

Eucelatoria teutonia Sabrosky

Euhuascaraya nemo Curran

Euoestrophasia plaumanni Guimaraes

Euoestrophasia townsendi Guimaraes

Jurinella bella Curran

Jurinella salla Curran

Jurinella vaga Curran

Leschenaultia aldrichi Toma \& Guimaraes

Leschenaultia bessi Toma \& Guimaraes

Leschenaultia coquilletti Toma \& Guimaraes

Lespesia plaumanni Guimaraes

Macromya ciniscula Reinhard

Miamimyia lopesi Guimaraes

Paratheresia cerambycivora Guimaraes

Paratheresia plaumanni Guimaraes

Polybiocyptera plaumanni Guimaraes

Tachinidae

Proparachaetopsis carvalhoi Toma \& Guimaraes

Thelairaporia brasiliensis Guimaraes

Triodontopyga vibrissata Guimaraes

Uramya insolita Guimaraes

Uramya plaumanni Guimaraes

Acaulona costata Wulp

Euacaulona sumichrasti Townsend

Itaxanthomelana grandis Townsend

Mahauiella nayrae Toma

Copecrypta nitens Wiedemann

Neocuphocera nepos Townsend

Deopalpus pictipennis Townsend

Archytas seminigra Wiedemann

Archytas sabroskyi Guimaraes

Archytas cirphis Curran

Archytas lopesi Guimaraes

Archytas lanei Guimaraes

Prophorostoma pulchra Townsend

Melaloncha diffidentia Brown

Dohrniphora anteroventralis Borgmeier

Phoridae

Tephritidae

Dohrniphora diplocantha Borgmeier

Dohrniphora gigantea Enderlein

Anastrepha aczeli Blanchard

Anastrepha amita Zucchi

Anastrepha barbielinii Lima

Anastrepha dissimilis Stone

Anastrepha distincta Greene

Anastrepha elegans Blanchard

Anastrepha fraterculus Wiedemann

Anastrepha grandis Macquart

Anastrepha montei Lima

\section{Ordem/ Família}

Espécie

Anastrepha obliqua Macquart

Anastrepha pseudoparallela Loew

Anastrepha similis Greene

Anastrepha sororcula Zucchi

Anastrepha xanthochaeta Hendel

Tephritidae

Blepharoneura poecilosoma Schiner

Ceratitis capitata Wiedemann

Dioxyna chilensis Macquart

Hexachaeta socialis Wiedemann

Rhagoletotrypeta pastranai Aczel

Tomoplagia sp.

\begin{tabular}{ll}
\hline Ulidiidae & Cymatosus polymorphomyiodes Enderlein \\
\cline { 1 - 1 } Asilidae & Ommatius orenoquensis Bigot \\
\cline { 1 - 2 } Sciomyzidae & Thecomyia limbata Wiedemann \\
\hline Otitidae & Euxesta sp. \\
\hline Therevidae & Psilocephala ornata Krober \\
\hline Dolichopodidae & Condylostylus sp. \\
\hline & Alluaudomyia catarinensis Spinelli \& Wirth \\
& Bezzia catarinensis Spinelli \& Wirth
\end{tabular}

Ceratopogonidae

Amerohelea pseudofasciata Grogan \& Wirth

Forcipomyia (Microhelea) penultimata

Alluaudomyia plaumanni Spinelli \& Wirth

Tipulidae

Ozodicera (Dihexacionus) macracantha Alexander

Austrolimnophila (Limnophilella) multipicta Alexander

Elephantomyia sp.

Limonia sp.

Limoniidae

Molophilus tridigitatus Alexander

Sigmatomera angustirostris Alexander

Molophilus subiratus Alexander

Limonia (Neolimnobia) archangelica Alexander

Molophilus phallosomicus Alexander

Somatiidae

Somatia australis Steyskal

Stratiomyidae

Artemita podexargenteus Enderlein

\section{Trichoptera}

Leptoceridae

Triplectides gracilis Burmeister

Nectopsyche fuscomaculata Flint

Smicridea piraya Flint

Hydropsychidae

Smicridea vermiculata Flint

Philopotamidae

Chimarra brasiliana Ulmer

Chimarra ypsilon Flint

Abstrichia sp.

Hydroptilidae

Neotrichia teutonia Flint

Neotrichia abbreviata Flint

Marilia flexuosa Ulmer

Odontoceridae

Marilia infundibulum Flint

Helicopsychidae 


\begin{tabular}{ll}
\hline \multicolumn{1}{c}{ Ordem/Família } & \multicolumn{1}{c}{ Espécie } \\
\hline Calamoceratidae & Phylloicus plaumanni Flint \\
\cline { 1 - 1 } Psychomyidae & Cernotina sp. \\
\cline { 1 - 1 } Glossosomatidae & Antoptila plaumanni Flint \\
\hline
\end{tabular}

\section{Lepidoptera}

\begin{tabular}{ll}
\hline Sematuriidae & Sematura cf. diana Gueneé \\
& Iridopsis $\mathrm{sp}$.
\end{tabular}

Geometridae

Pantherodes pardalaria Hübner

Cundinamarca rindgei Dias

Actinote melanisans Oberthür

Actinote hyalina Brown

Actinote carycina Jordan

Adelpha zea Hewitson

Adelpha serpa Boidusval

Adelpha syma Godart

Adelpha abia Hewitson

Adelpha hyas Doyère

Adelpha mythra Godart

Adelpha thessalia C. \& R. Ferlder

Agraulis sp.

Anartia amathea Linnaeus

Archaeoprepona chalciope Hubner

Brassolis astyra Godart

Brassolis sophorae Linnaeus

Biblis hyperia Cramer

Caligo martia Godart

Caligo illioneus Cramer

Catoblepia berecynthia Cramer

Chlosyne lacinia Geyer

Danaus erippus Cramer

Nymphalidae

Danaus cleophille Godart

Dasyophthalma creusa Hübner

Diaethria clymena Cramer

Dircenna dero Hübner

Dynamine tithia Hübner

Dynamine mylitta Cramer

Dynamine myrrhina Doubleday

Dynastor darius Fabricius

Doxocopa laurentia Godart

Epityches eupompe Geyer

Episcada carcinia Schaus

Episcada hymeneae Prittwitz

Eresia lansdorfi Godart

Eueides isabella Stoll

Euptychia sp.

Eryphanis reevesii Doubleday

Hamadryas amphinome Linnaeus

Hamadryas epinome Felder \& Felder

Hamadryas februa Hübner

Hamadryas formax Hübner

Heliconius erato Linnaeus

\begin{tabular}{|l|l|}
\hline Ordem/ Família & \multicolumn{1}{c}{ Espécie } \\
\hline Heliconius besckei Ménétriés \\
\hline Hypanartia sp. \\
\hline Hypolimnas misippus Linnaeus \\
\hline Junonia evarete Cramer \\
\hline Lycorea cf. cleobaea Godart \\
\hline Lycorea ilione Cramer \\
\hline Manataria hercyna Hübner \\
\hline Marpesia petreus Cramer \\
\hline Mechanitis lysimnia Fabricius \\
\hline Memphis moruus Fabricius \\
\hline Methona themisto Hübner \\
\hline Morpho cf. achilles Linnaeus \\
\hline Morpho catenarius Perry \\
\hline Morpho menelaus Linnaeus \\
\hline Morpho aega Hübner \\
\hline Myscelis orsis Drury \\
\hline Narope sp. \\
\hline Opsiphanes batea Hübner \\
\hline Opsiphanes invirae Hübner \\
\hline Opsiphanes quiteria Stoll \\
\hline Ortilia orthia Hewitson \\
\hline Ortilia ithra Kirby \\
\hline Ortilia dicoma Hewitson \\
\hline Nymphalidae
\end{tabular}

Paulogramma pyracmon Godart

Penetes pamphanis Doubleday

Placidula euryanassa C. \& R. Felder

Pseudoscada erruca Hewitson

Pteronymia sp.

Siproeta trayja Hübner

Siproeta stelenes Linnaeus

Tegosa claudina Eschscholtz

Zaretis isidora Cramer

Pterourus scamander Boisduval

Pterourus menatius Hopffer

Heraclides androgeus Cramer

Heraclides hectorides Esper

Heraclides astyalus astyalus Godart

Heraclides anchisiades capys Hübner

Heraclides thoas brasiliensis Rothschild \& Jordan

Papilionidae

Battus polydamas polydamas Linnaeus

Battus polystictus Butler

Mimoides lisithous eupatorion Lucas

Parides agavus Drury

Parides anchises Linnaeus

Parides bunichus perrhebus Boisduval

Protesilaus protesilaus Linnaeus

Protesilaus stenosdesmus Rothschild \& Jordan

Almeidella approximans Schaus

Saturniidae

Almeidella corrupta Schaus

Arsenura orbignyana Guérin-Meneville 


\section{Ordem/ Família}

Arsenura armida Cramer

Automerella flexuosa Felder

Automerella aurora Massen \& Weiding

Automeris cf. naranja Schaus

Automeris illustris Walker

Automeris basalis Walker

Automeris inornata Walker

Copaxa sp.

Copaxa decrescens Walker

Copaxa flavina Draudt

Copaxa multifenestrata Herrich-Schäffer

Copiopteryx jehovah Strecker

Citheronia laocoon Cramer

Citioica anthonilis Herrich-Schäffer

Dirphia sp.

Dirphia araucariae Jones

Dirphia ursina Walker

Eacles imperialis magnifica Walker

Eacles penelope Cramer

Hyperchiria incisa Walker

Saturniidae

\section{Leucanella sp.}

Leucanella memusae Walker

Lonomia sp.

Lonomia electra Druce

Molippa sp.

Molippa cruenta Walker

Neocarnegia basirei Schaus

Oiticella brevis Walker

Oiticella convergens Herrich-Schäffer

Othorene purpurascens Schaus

Paradaemonia sp.

Pseudautomeris coronis Schaus

Rothschildia arethusa Walker

Rothschildia aurota Cramer

Rothschildia jacobeae Walker

Rothschildia hoppferi Felder \& Felder

Scolesa totoma Schaus

Adeloneivaia sp.

Adelowalkeria flavosignata Walker

Syssphinx molina Cramer

Travassosula subfumata Schaus

Adhemarius eurysthenes Felder

Aellopos titan Cramer

Callionimia cf. parce Fabricius

Cocytius lucifer Rothschild \& Jordan

Sphingidae

Erinnyis ello Linnaeus

Eumorpha satellitia Linnaeus

Manduca sp.

Nyceryx continua Walker

Callionima pan Cramer
Ordem/ Família

Espécie

Chlaenogramma muscosa Jones

Cocytius antaeus Drury

Enyo gorgon Cramer

Erinnyis obscura Fabricius

Manduca albiplaga Walker

Pachylia ficus Linnaeus

Sphingidae

Manduca armatipes Rothschild \& Jordan

Phryxus caicus Cramer

Xylophanes isaon Boisduval

Xylophanes chiron nechus Cramer

Xylophanes pistacina Boisduval

Xylophanes tyndarus Boisduval

Ascia monuste Linnaeus

Phoebis sp.

Pieris sp.

Eurema sp.

Pieridae

Dismorphia astyocha Hübner

Dismorphia melia Godart

Dismorphia thermesia Godart

Enantia melite Linnaeus

Urbanus sp.1

Urbanus sp. 2

Urbanus sp. 3

Hesperiidae

Urbanus sp. 4

Lychnuchoides ozias Hewitson

Lycas argentea Hewitson

Trina sp.

Dysschema sacrifica Hübner

Arctiidae

Hypocrita bicolora Sulzer

Phaloe cruenta Hübner

Utetheisa ornatrix Linnaeus

Chorinea cf. licursis Fabricius

Caria sp.

Adelotypa sp.

Eurybia pergaea Geyer

Riodinidae

Melanis xenia Hewitson

Rhetus periander Cramer

Riodina lycisca Hewitson

Synargis calyce C. Felder \& R. Felder

Ascalapha odorata Linnaeus

Eudocima sp.

Noctuidae

Cirphis hildrani Schaus

Spodoptera frugiperda Smith

Helicoverpa zea Boddie

Diatraea sp.

Eldana saccharina Walker

Volatica hemirhodella Hampson

Diptychophora kuhlweini Zeller

Argyrotaenia sphaleropa Meyrick Orthocomotis twila Clarke

Continua..

Tortricidae 


\begin{tabular}{ll}
\hline \multicolumn{1}{c}{ Ordem/ Família } & \multicolumn{1}{c}{ Espécie } \\
\hline \multirow{4}{*}{ Tortricidae } & Eulia deceptiva Clarke \\
& Polyortha myoxa Razowski \\
& Eulia episticta Clarke \\
& Eulia virga Clarke \\
& Orthocomotis mareda Clarke \\
& Cochylis serena Clarke \\
& Dimorphopalpa teutoniana Brown \\
& Melittia umbrosa Zukowsky \\
\hline Sesiidae & Coptotelia complicata Clarke \\
\hline Oecophoridae & Gonionota argopleura Clarke \\
& Gonionota selene Clarke \\
\hline & Hymenoptera \\
\hline
\end{tabular}

Formicidae

\section{Hymenoptera}

Ceraphacyinae

\begin{tabular}{ll}
\hline & Acanthostichus flexuosus Mackay \\
\hline Acanthostichini & Acanthostichus quadratus Emery \\
& Acanthostichus serratulus Smith \\
\hline Cerapachyini & Cerapachs splendens Borgmeier
\end{tabular}

Cerapachyini Sphinctomyrmex stali Mayr

Dolichoderinae

Dorymyrmex brunneus Forel

Linepithema bruchi Santschi

Linepithema humile Mayr

Dolichoderini

Linepithema iniquum Mayr

Linepithema leucomelas Emery

Tapinoma atriceps Emery

Tapinoma melanocephalum Fabricius

Ecitoninae

Eciton quadriglume Haliday

Eciton burchellii Westwood

Labidus coecus Latreille

Ecitonini

Labidus praedator Smith

Neivamyrmex hetschkoi Mayr

Neivamyrmex punctaticeps Emery

Neivamyrmex tenuis Borgmeier

Formicinae

mex tenuis Borgmeier

\begin{tabular}{ll}
\hline Brachymyrmex (Brachymyrmex) coactus Mayr \\
\hline Brachymyrmex (Brachymyrmex) cordemoyi Forel \\
Brachymyrmecini & $\begin{array}{l}\text { Brachymyrmex (Brachymyrmex) heeri aphidicola } \\
\text { Forel }\end{array}$ \\
\hline Brachymyrmex (Brachymyrmex) pilipes Mayr & Brachymyrmex (Brachymyrmex) santschii Menozzi \\
\hline Camponotus (Hypercolobopsis) paradoxos & Camponotus (Myrmobrachys) caracalla Forel \\
\hline Camponotus (Myrmobrachys) crassus Mayr \\
\hline Camponotus (Myrmobrachys) mus Roger \\
\hline Camponotus (Myrmocladoecus) hedwigae Forel \\
\hline Camponotus (Myrmothrix) rufipes Fabricius \\
\hline Camponotus (Pseudocolobopsis) alboannulatus Mayr \\
\hline Camponotus (Pseudocolobopsis) macrocephalus \\
Erichson
\end{tabular}

\section{Ordem/ Família}

Espécie

Camponotus (Tanaemyrmex) bonariensis garbei Santschi

Camponotus (Tanaemyrmex) bonariensis parvulus

Emery

Camponotus (Tanaemyrmex) fuscocinctus Emery

Camponotini

Camponotus (Tanaemyrmex) melanoticus Emery

Camponotus (Tanaemyrmex) pallescens Mayr

Camponotus (Tanaemyrmex) simillimus Smith

Camponotus (Tanaemyrmex) xanthogaster Santschi

Camponotus (Tanaemyrmex) zenon Forel

Myrmelachista bambusarum Forel

Myrmelachista catharinae Mayr

Myrmelachista kloetersi Forel

Myrmelachistini

Myrmelachista nodigera Mayr

Myrmelachista reticulata Borgmeier

Myrmelachista ruszkii Forel

Myrmicinae

Acromyrmex (Acromyrmex) aspersus Smith

Acromyrmex (Acromyrmex) crassispinus Forel

Acromyrmex (Acromyrmex) hispidus Santschi

Acromyrmex (Acromyrmex) laticeps Emery

Acromyrmex (Acromyrmex) subterraneus brunneus

Forel

Apterostigma moelleri Forel

Apterostigma pilosum Mayr

Apterostigma wasmanni Forel

Atta (Neoatta) sexdens Linnaeus

Attini

Cyphomyrmex hamulatus Weber

Cyphomyrmex occultus Kempf

Cyphomyrmex olitor Forel

Cyphomyrmex peltatus Kempf

Cyphomyrmex plaumanni Kempf

Cyphomyrmex rimosus Spinola

Mycetarotes senticosus Kempf

Mycetosoritis aspera Mayr

Mycocepurus goeldii Forel

Myrmicocrypta bruchi Santschi

Basiceros disciger Mayr

Eurhopalothrix spectabilis Kempf

Eurhopalothrix speciosa Brown \& Kempf

Basicerotini

Octostruma rugifera Mayr

Octostruma stenognatha Brown \& Kempf

Rhophalothrix sp. 1

Rhophalothrix sp. 2

Procryptocerus adlerzi Mayr

Procryptocerus convergens Mayr

Procryptocerus goeldii Forel

Procryptocerus lenkoi Kempf

Procryptocerus lepidus Forel

Procryptocerus regularis Emery

Procryptocerus schmalzi Emery 


Ordem/Família $\quad$ Espécie

Zacryptocerus angustus Mayr

Zacryptocerus depressus Klug

Cephalotini

Zacryptocerus sp.

Zacryptocerus pusillus Klug

Zacryptocerus striativentris Emery

Crematogaster (Eucrema) acuta Fabricius

Crematogaster (Eucrema) bingo Forel

Crematogaster (Neocrema) corticicola Mayr

Crematogaster (Neocrema) magnifica Santschi

Crematogastrini

Crematogaster (Orthocrema) brevispinosa moelleri

Forel

Crematogaster (Orthocrema) crinosa Mayr

Crematogaster (Orthocrema) curvispinosa Mayr

Crematogaster (Orthocrema) lutzi Forel

Acanthognathus ocellatus Mayr

Acanthognathus rudis Brown \& Kempf

Glamyromyrmex appretiatus Borgmeier

Gymnomyrmex minusculus Kempf

Gymnomyrmex rugithorax Kempf

Gymnomyrmex splendens Borgmeier

Neostruma crassicornis Kempf

Dacetonin

Phalacromyrmex fugax Kempf

Smithistruma tanymastax Brown

Strumigenys cordovensis Mayr

Strumigenys cultriger Mayr

Strumigenys denticulata Mayr

Strumigenys louisianae Roger

Strumigenys saliens Mayr

Strumigenys silvestrii Emery

Leptothorax (Nesomyrmex) schwebeli Forel

Formicoxenini

Leptothorax (Nesomyrmex) vicinus Mayr

Myrmicini

Hylomyrma balzani Emery

Hylomyrma reitteri Mayr

Pheidole (Elasmopheidole) aberrans Mayr

Pheidole (Pheidole) auropilosa Mayr

Pheidole (Pheidole) bambusarum Forel

Pheidole (Pheidole) brevicornis Mayr

Pheidole (Pheidole) dyctiota Kempf

Pheidole (Pheidole) flavens Roger

Pheidole (Pheidole) guilelmimuelleri Forel

Pheidole (Pheidole)hetschkoi Emery

Pheidolini

Pheidole (Pheidole) impariceps Santschi

Pheidole (Pheidole) nana Emery

Pheidole (Pheidole) pubiventris Mayr

Pheidole (Pheidole) punctatissima Mayr

Pheidole (Pheidole) risii Forel

Pheidole (Pheidole) subarmata Mayr

Pheidole (Pheidole) tristis Smith

Pheidole (Trachypheidole) aper Forel

\begin{tabular}{ll}
\hline \multicolumn{1}{c|}{ Ordem/ Família } & \multicolumn{1}{c}{ Espécie } \\
\hline Pheidologetonini & Carebara mayri Forel \\
\hline Ochetomyrmicini & Wasmmania auropunctata Roger \\
\hline & Megalomyrmex drifti Kempf \\
& Megalomyrmex myops Santschi \\
& Megalomyrmex pusillus Forel \\
& Megalomyrmex silvestrii Wheeler \\
& Megalomyrmex sp. \\
\hline Solenopsidini & Oxyepoecus crassinodus Kempf \\
& Oxyepoecus plaumanni Kempf \\
& Oxyepoecus punctifrons Borgmeier \\
& Oxyepoecus rastratus Mayr \\
& Oxyepoecus reticulatus Kempf \\
& Oxyepoecus vezenyii Forel \\
\hline Stenammini & Lachnomyrmex plaumanni Borgmeier \\
\hline Ponerinae & Rogeria pellecta Kempf \\
\hline
\end{tabular}

Amblyopone armigera Mayr

Amblyoponini

Amblyopone degenerata Borgmeier

Amblyopone elongata Santschi

Acanthoponera goeldii Forel

Acanthoponera mucronata Roger

Gnamptogenys lucaris Kempf

Gnamptogenys minuta Emery

Gnamptogenys moelleri Forel

Gnamptogenys rastrata Mayr

Gnamptogenys reichenspergeri Santschi

Ectatommini

Gnamptogenys striatula Mayr

Gnamptogenys striolata Borgmeier

Gnamptogenys triangularis Mayr

Heteroponera dolo Roger

Heteroponera inermis Emery

Heteroponera mayri Kempf

Heteroponera microps Borgmeier

Anochetus altisquamis Mayr

Dinoponera australis Borgmeier

Hypoponera collegiana Santschi

Hypoponera distinguenta Emery

Hypoponera foeda Forel

Hypoponera foreli Mayr

Hypoponera opacior Forel

Ponerini

Hypoponera reichenspergeri Santschi

Hypoponera schwebeli Forel

Hypoponera trigona Mayr

Hypoponera wilsoni Santschi

Leptogenys (Lobopelta) australis Emery

Odontomachus chelifer Latreille

Pachycondyla crenata Roger

Continua... 


\begin{tabular}{ll}
\hline \multicolumn{1}{c}{ Ordem/ Família } & \multicolumn{1}{c}{ Espécie } \\
\hline Ponerini & Pachycondyla ferruginea Smith \\
& $\begin{array}{l}\text { Pachycondyla harpax } \text { Fabricius } \\
\text { Pachycondyla striata } \text { Smith }\end{array}$ \\
\hline Proceratiini & Discothyrea neotropica Bruch \\
\hline Thaumatomyrmecini & Discothyrea sexarticulata Borgmeier \\
\hline Typhlomyrmicini & Thaumatomyrmex mutilatus Mayr \\
\hline Pseudomyrmecinae & Typhlomyrmex pusillus Emery \\
\hline
\end{tabular}

\section{Ordem/ Família}

\begin{tabular}{lr}
\hline Ordem/ Família & Espécie \\
\hline & Plebeia remota Holmberg
\end{tabular}

Plebeia saiqui Hohnberg

Plebeia nigriceps Friese

Plebeia emerina Friese

Nannotrigona testaceicornis Lepeletier

Lestrimelitta sp.

Apini

Scaptotrigona bipunctata Lepeletier

Scaptotrigona depilis Moure

Schwarziana quadripunctata Lepeletier

Tetragonisca angustula Latreille

Trigona spinipes Fabricius

Tetragona clavipes Fabricius

Cephalotrigona capitata Smith

Brachynomadini

Brachynomada sp. Holmberg

Andrenidae

Acamptopoeum prinii Holmberg

Calliopsini

Callonychium petuniae Cure \& Wittman

Calliopsis sp.

Protandrena (Heterosarus) sp.

Anthrenoides sp. 1

Anthrenoides sp. 2

Anthrenoides araucariae Urban

Anthrenoides meridionalis Schrottky

Anthrenoides paolae Urban

Parapsaenythia serripes Ducke

Protandrenini

\section{Psaenythia anullata Gerstaecker}

Psaenythia bergi Holmberg

Psaenythia capito Gerstaecker

Psaenythia quadrifasciata Friese

Rhophitulus anomalus Moure \& Lucas de Oliveira

Rhophitulus flavitarsis Schlindwein \& Moure

Rhophitulus reticulatus Schlindwein \& Moure

\section{Apidae}

Osirini

Osiris variegatus Smith

Apis mellifera Linnaeus

Bombus sp. 1

Bombus atratus Franklin

Bombus morios Swederus

Eufriesea violacea Blanchard

Melipona (Eomelipona) marginata Lepeletier

Melipona obscurior Moure

Apini

Centridini

Centris (Hemisiella) tarsata Smith

Centris (Trachina) proxima Friese

Centris fuscata Lepeletier

Centris varia Erichson

Epicharis (Epicharoides) grandior Friese

Ceratina (Ceratinula) biguttulata Moure

\begin{tabular}{l} 
Ceratinini \\
\hline Emphorini \\
\hline Epeolini \\
\hline
\end{tabular}

Ceratina (Crewella) cf. asuncionis Strand

Melitoma segmentaria Fabricius

Trophocleptria cf. variolosa Holmberg

Ericrocidini

Mesocheira bicolor Fabricius

Mesoplia sp.

Melissodes (Ecplectica) tintinnans Holmberg

Melissodes (Ecplectina) nigroaenea Smith

Melissodes (Ecplectina) sexcincta Lepeletier

Melissoptila bonaerensis Holmberg

Melissoptila aureocincta Urban

Melissoptila cnecomala Moure

Eucerini

Melissoptila larocai Urban

Melissoptila marinonii Urban

Melissoptila minarum Bertoni \& Schrottky

Peponapis fervens Smith

Thygater analis Lepeletier

Ptilomelissa leucozonata Zikán \& Wygodzinsky

Exomalopsis (Exomalopsis) analis Spinola

Exomalopsini

Exomalopsis (Exomalopsis) tomentosa Friese

Exomalopsis (Phanomalopsis) trifasciata Brèthes

Doeringiella (Orfilana) cingillata Moure

Nomadini

Nomada costalis Brèthes

Epeolus sp.

Arhrysoceble picta Friese

Chalepogenus sp.

Tapinotaspidini

Lanthanomelissa betinae Urban

Paratetrapedia fervida Smith 


\begin{tabular}{ll}
\hline \multicolumn{1}{c}{ Ordem/ Família } & \multicolumn{1}{c}{ Espécie } \\
\hline Tapinotaspidini & Paratetrapedia (Lophopedia) cf. nigrispinnis Vachal \\
\hline Tetrapedini & Tetrapedia diversipes Klug \\
\hline & Xylocopa (Neoxylocopa) augusti Lepeletier \\
& Xylocopa (Neoxylocopa) frontalis Olivier \\
& Xylocopa (Stenoxylocopa) artifex Smith \\
Xylocopini & Xylocopa (Nanoxylocopa) ciliata Burmeister \\
& Xylocopa plaumanni Moure \\
\hline Tapinotaspidini & Ceratina sp. \\
\hline Colletidae & Lanthanomelissa betinae Urban \\
\hline Colletini & Lanthanomelissa clementis Urban \\
\hline Hylaeini & Colletes rugicolis Friese \\
\hline & Hylaeus sp. \\
\hline Paracolletini & Belopria sp. \\
\hline Halictidae & Hexantheda missionica Oglobin \\
\hline & Perditomorpha leaena Vachal \\
\hline & Tetraglossula anthracina Michener \\
\hline & Leioproctus sp. \\
\hline
\end{tabular}

Halictidae
\begin{tabular}{ll}
\hline Ariphanarthra palpalis Moure \\
\hline Augochlora amphitrite Schrottky \\
\hline Augochlora aff. semiramis Schrottky \\
\hline Augochlora foxiana Cockerell \\
\hline Augochlora cyphogastra Moure \\
\hline Augochlora nitidior Moure \\
\hline Augochlorella ephyra Schrottky \\
\hline Augochlorella urania Smith \\
\hline Augochlorella iopaecila Moure \\
\hline Augochloropsis cupreola Cockerell \\
\hline Ceratalictus sp. \\
\hline Corynura sp.
\end{tabular}

Augochlorini

Pseudagapostemon cyanomelas Cure

Pseudagapostemon cyaneus Moure \& Sakagami

\section{Ordem/ Família}

Espécie

Anthidium manicatum Linnaeus

Anthidiellum sp.

Anthodioctes claudii Urban

Anthodioctes misiutae Urban

Anthodioctes megachiloides Holmberg

Austrostelis iheringii Schrottky

Carloticola paraguayensis Schrottky

Epanthidium autumnale Schrottky

Anthidini

Epanthidium bicoloratum Smith

Epanthidium paraguayensis Schrottky

Hypathidium divaricatum Smith

Hypanthidioides (Saranthidium) musciforme Schrottky

Hypanthidioides sp.

Moureanthidium catarinense Urban

Moureanthidium paranaense Urban

Saranthidium musciforme Schrottky

Gnathanthidium sakagamii Urban

Coelioxys (Acrocoelioxys) tolteca Cresson

Coelioxys (Cyrtocoelioxys) cf. dobzhanskyin Moure

Coelioxys (Glyptocoelioxys) labiosa Moure

Coelioxys (Cyrtocoelioxys) aff. quaerens Holmberg

Megachile (Acentron) lentifera Vachal

Megachile (Austromegachile) sussurrans Haliday

Megachilini

Megachile (Crysosarus) sp.

Megachile (Dactylomegachile) sp.

Megachile (Leptorachis) aetheria Mitchell

Megachile (Moureapis) anthidioides Radozkowsky

Megachile (Moureapis) apicipennis Schrottky

Megachile (Moureapis) cf. nigropilosa Schrottky

Megachile (Pseudocentron) sp.

Rhabdepyris (Chlorepyris) vesculus Evans

Rhabdepyris (Chlorepyris) virescens Evans

Bethylidae

Rhabdepyris (Rhabdepyris) nigriscapus Evans

Epyris depressigaster Urban

Epyris sp.

Notiospathius atra De Jesús-Bonilla, Nunes, Penteado-Dias, Zaldívar-Riverón

Notiospathius johnlennoni De Jesús-Bonilla, Nunes, Penteado-Dias, Zaldívar-Riverón

Notiospathius novateutoniae De Jesús-Bonilla, Nunes, Penteado-Dias, Zaldívar-Riverón

Notiospathius sulcatus De Jesús-Bonilla, Nunes, Penteado-Dias, Zaldívar-Riverón

Braconidae

Notiospathius xanthofasciatus De Jesús-Bonilla, Nunes, Penteado-Dias, Zaldívar-Riverón

Meteoridea whartoni Penteado-Dias

Meteoridea achterbergi Penteado-Dias

Aleoides flavistigma Shaw

Trachagathis pengellyella Sharkey

Chrysididae Neochrysis bubba Kimsey 


\begin{tabular}{ll}
\hline \multicolumn{1}{c}{ Ordem/ Família } & \multicolumn{1}{c}{ Espécie } \\
\hline Eulophidae & Acanthala plaumanni Hansson \\
& Podkova sp. \\
& Jomine una Graf \& Kumagai \\
& Labena fiorii Graf \& Marzagao \\
Ichneumonidae & Xorides (Pyramirhyssa) magnificus Mocsary \\
& Notocampis sp. \\
\hline Monomachidae & Monomachus aurifer Musetti \& Johnson \\
\hline Pompilidae & Aridestus bergi Brèthes \\
\hline Scelionidae & Pepsis sp. Fabricius \\
\hline Sphecidae & Telenomus angulatus Johnson \\
& Oxybelus genisei Bohart \\
\hline
\end{tabular}

\section{AGRADECIMENTOS}

Os autores são gratos à Cláudia Fontana pela ajuda com a definição das formações florestais da região oeste de Santa Catarina e aos revisores da EntomoBrasilis por todas as sugestões e correções.

\section{REFERÊNCIAS BIBLIOGRÁFICAS}

Allen, R.K., 1967. New species of New World Leptohyphinae (Ephemeroptera: Tricorythidae). Canadian Entomologist, 99: 350-375.

Ale-Rocha, R., 2004. Revisão das espécies sul-americanas de Euhybus (Diptera, Hybotidae, Hybotinae) do grupo purpureus. Iheringia, Série Zoologia, 94: 357-373.

Almeida, L.M. \& I.M.M. Lima, 1995. Revisão do gênero Oryssomus Mulsant (Coleoptera, Coccinellidae, Exoplectrinae, Oryssomini) e descrição de Gordonoryssomus, gen.n. Revista Brasileira de Zoologia, 12: 701-718.

Aragão, A.C. \& M.A. Monné, 2011. O gênero Megacyllene s.str. Casey (Coleptera, Cerambycidae) na Mata Atlântica: descrição de duas espécies inéditas, chave para identificação e novas ocorrências. Revista Brasileira de Entomologia, 55: 159-171.

Araújo-Siqueira, M. \& L.M. Almeida, 2006. Estudo das espécies brasileiras de Cycloneda Crotch (Coleoptera, Coccinellidae). Revista Brasileira de Zoologia, 23: 550-568.

Bequart, J., 1943. Notes on Hippoboscidae. 16. Hippoboscidae from southern Brazil, with the description of a new specie of Lynchia. Journal of Parasitology, 29: 131-135.

Bicho, C.A. \& L.M. Almeida, 1998. Revisão do gênero Neocalvia Crotch (Coleoptera, Coccinellidae). Revista Brasileira de Zoologia, 15: 167-189.

Bohart, R.M.,1993. South American Oxybelus II. The emarginatus group (Hymenoptera: Sphecidae). Insecta Mundi, 7: 18-26.

Borowiec, L., 2002. New records of Neotropical Cassidinae, with description of three new species (Coleoptera: Chrysomelidae). Genus, 13: 43-138.

Bremer, H.J. \& C.A. Tirplerhorn, 1999. The Latin American species of the genus Corticeus Piller and Mitterpacher (Coleoptera, Tenebrionidae, Hypophloeini). Part I. The species described by Reitter and Pic, and description of two new species. The Coleopterists Bulletin, 53: 56-63.

Camras, S., 1957. Descriptions and records of Neotropical Conopidae (Diptera). Psyche, 64: 9-16.

Carvalho, C.J.B., 2012. Biodiversidade e Conservação, p. 133-138 In: Rafael, J.A., G.A.R. Melo, C.J.B. de Carvalho, S.A. Casari \& R. Constantino (Eds.). Insetos do Brasil: Diversidade e Taxonomia. Ribeirão Preto: Holos, 796p.

Caterino, M.S. \& A.K. Tishechkin, 2008. A review of Hippeutister Reichensperger with new species from California and Costa Rica (Coleoptera, Histeridae, Hetaeriinae). Zootaxa, 1895: 39-52.
Ordem/ Família

Espécie

\begin{tabular}{ll}
\hline \multicolumn{1}{c|}{ Ordem/ Família } & \multicolumn{1}{c}{ Espécie } \\
\hline Encyrtidae & Arhopoidiella carinata Noyes \\
& Lirencyrtus primus Noyes \\
& Neapsilophrys flavipes Noyes \\
& Papaka confusor Noyes \\
\hline Vespidae & Cephalastor bossanova Garcet-Barret \\
& Paramasaris brasiliensis Soika \\
\hline Cimcibidae & Polistes cavapyta Saussure \\
\hline Tiphiidae & Ceratina sp. \\
\hline Orussidae & Catocheilus sp. \\
\hline Tenthredinidae & Ophrynopus depressatus Smith \\
\hline
\end{tabular}

Clarke, R.O.S., U.R. Martins \& A. Santos-Silva, 2011. Contribuição para o estudo de Rhinotragini (Coleptera, Cerambycidae). IV. Rhopalessa Bates, 1873. Papéis Avulsos de Zoologia, 51: 325339.

Coelho, S.M.P., C.J.B. Carvalho \& J.H. Guimarães, 1989. Chave e sinonímias para as espécies sul-americanas de Winthemia Robineau-Desvoidy (Diptera, Tachinidae) com descrição de três novas espécies. Revista Brasileria de Zoologia, 6: 271296.

Costa-Lima, A.M., 1945. Insetos do Brasil: Lepidópteros. $5^{\circ}$ Tomo. Escola Nacional de Agronomia, Série Didática N. ${ }^{\circ} 7$. 376p.

Costa-Lima, A.M., 1950. Insetos do Brasil: Lepidópteros-2ª parte. $6^{\circ}$ Tomo. Escola Nacional de Agronomia. Série Didática N. ${ }^{\circ}$ 8. $414 \mathrm{p}$.

Costa, A.V., L.M. Almeida \& G.H. Corrêa, 2008. Revisão das espécies brasileiras do gênero Exoplectra Chevrolat (Coleoptera, Coccinellidae, Exoplectrinae, Exoplectrini). Revista Brasileira de Entomologia, 52: 365-383.

Couri, M.S., J.L. Nessimian, G. Mejdalani, M.L. Monné, S.M. Lopes, M.C. Mendonça, R. Monteiro, S. Buys \& R.A. Carvalho, 2009. Levantamento dos insetos da Mata Atlântica do Estado do Rio de Janeiro. Arquivos do Museu Nacional, 67: 151-154.

DeLong, D.M., 1980a. New species of Central and South American Gypona (Homoptera: Cicadellidae). Brenesia, 17: 215-250.

DeLong, D.M., 1980b. New species of Gypona (Homoptera, Cicadellidae, Gyponinae) from Central and South America. Revista Peruana de Entomologia, 23: 59-62.

Dias, M.M., 1998. Considerações taxonômicas sobre o gênero Cundinamarca Rindge (Lepidoptera, Geometridae) e descrição de uma nova espécie. Revista Brasileira de Zoologia, 15: 951-958.

Domínguez, E., 1995. Cladistic analysis of the UlmeritusUlmeritoides group (Ephemeroptera, Leptophlebiidae), with descriptions of five new species of Ulmeritoides. Journal of the New York Entomological Society, 103: 15-38.

Duarte, M., G. Marconato, A. Specht, M.M. Casagrande, 2012. Lepidoptera. p. 625-684. In: Rafael, J.A., G.A.R. Melo, C.J.B. Carvalho, S.A. Casari \& R. Constantino (Eds.). Insetos do Brasil: Diversidade e Taxonomia. Ribeirão Preto: Holos, 796p.

Evans, H.E., 1965. Further studies on Neotropical Epyrini (Hymenoptera, Bethylidae). Psyche, 72: 265-278.

Evans, H.E., 1966. Studies on Neotropical Pompilidae (Hymenoptera) II. Genus Aridestus Banks. Psyche, 73: 116122.

Favretto, M.A., 2012. Borboletas e mariposas (Insecta: Lepidoptera) do município de Joaçaba, Santa Catarina, Brasil. EntomoBrasilis, 5: 167-169.

Ferreia, P.S.F. \& T.J. Henry, 2010. Revision of the genus Ambracius Stal, 1860 (Heteroptera: Miridae: Deraeocorinae: 
Clivinematini), with description of three new species. Zootaxa, 2485: 1-15.

Fisher, W.S., 1952. New cerambycid beetles belonging to the tribe Rhinotragini from South America (Coleoptera). American Museum Novitates, 1552: 1-18.

Fisher, W.S., 1947. New cerambycid beetles belonging to the tribe Rhinotragini from South America. American Museum Novitates, 1349: 1-6.

Fluke, C.L., 1937. New South American Syrphidae (Diptera). American Museum Novitates, 941: 1-14.

Fluke, C.L., 1945. The Melanostomini of the Neotropical region (Diptera, Syrphidae). American Museum Novitates, 1272: 1-29.

Fonseca, C.R.V. \& M.F. Vieira, 2000. Descrição de três novas espécies do gênero Melittoma (Coleoptera, Lymexylidae) da região Neotropical e considerações sobre Melittoma brasiliense (Castelnau, 1832). Acta Amazonica, 31: 91-107.

Fortes, N.D.F. \& J. Grazia, 2005. Revisão e análise cladística de Serdia Stal (Heteroptera, Pentatomidae, Pentatomini). Revista Brasileira de Entomologia, 49: 294-339.

Froehlich, C.G., 2011. Notes on Kempnyia, with description of three new species (Plecoptera: Perlidae). Illiesia, 7: 133-141.

Galileo, M.H.M. \& U.R. Martins, 2005. Novas espécies e novas ocorrências de Xenofrea (Coleoptera, Cerambycidae, Lamiinae). Iheringia, Série Zoologia, 95: 383-388.

Galileo, M.H.M. \& U.R. Martins, 2009. Aleiphaquilon Martins (Coleoptera, Cerambycidae, Cerambycinae, Neocorini): novas ocorrências, nova espécie e chave. Papéis Avulsos de Zoologia, 49: 339-342.

Galileo, M.H.M. \& U.R. Martins, 2001. Novas espécies de Lamiinae (Coleoptera, Cerambycidae) neotropicais. Iheringia, Série Zoologia, 90: 59-70.

Garcia, F.R.M., Nardi, N., Costa, M.K.M. \& D.A. Brescovit, 2004. Ocorrência de artrópodes em lavoura de milho (Zea mays) no município de Arvoredo, SC. Bioikos, 18: 21-28.

Garcia, F.R.M., J.V. Campos \& E. Corseuil, 2003. Análise faunística de espécies de moscas-das-frutas (Diptera: Tephritidae) na região oeste de Santa Catarina. Neotropical Entomology, 32: 421-426.

Geller-Grimm, F., 1997. A new species of Ichneumolaphria Carrera from Brazil and the description of the male of Pseudorus dandrettae Carrera, 1949 (Diptera, Asilidae). Studia Dipterologica, 4: 457-162.

Gerdes, C.F., 1984. Eurythrips and Terthrothrips (Thysanoptera: Phlaeothripidae) from southern Brazil, with one new species, new collection sites, and key. Proceedings of Entomological Society of Washington, 86: 400-410.

Gomes, A.C., M.B. Paula, J.B.V. Neto, R. Borsari \& A.S. Ferraudo, 2009. Culicidae (Diptera) em Área de Barragem em Santa Catarina e no Rio Grande do Sul. Neotropical Entomology, 38: 553-555.

Graciani, C., F.R.M. Garcia \& M.K.M. Costa, 2005. Análise faunística de gafanhotos (Orthoptera, Acridoidea) em fragmentos florestal próximo ao rio Uruguai, município de Chapecó, Santa Catarina. Biotemas, 18: 87-98.

Graciolli, G., 2003. Nova espécie de Anatrichobius Wenzel, 1966 (Diptera, Streblidae) do Brasil meridional. Revista Brasileira de Entomologia, 47: 55-58.

Graf, V. \& A.F. Fumagai, 1997. Novo gênero de Campopleginae (Hymenoptera, Ichneumonidae) do Brasil. Revista Brasileira de Zoologia, 14: 649-658.

Graf, V. \& A.F. Fumagai, 2002. Notocampsis Townes (Cryptinae, Ichneumonidae, Hymenoptera) do Paraná, Brasil. Revista Brasileira de Zoologia, 19: 1177-1180.

Graf, V., 1995. Nota sobre Xorides Latreille (Ichneumonidae, Hymenoptera) no Brasil. Revista Brasileira de Zoologia, 12: 31-35.

Graf, V. \& M.R. Marzagão, 1999. Ocorrência do parasitóide Labena fiorii sp.n. (Hymenoptera, Ichneumonidae) em larvas de Hedypathes betulinus (Klug), broca da erva-mate e em Chydarteres striatus (Fabricius), broca da aroeira,
(Coleoptera, Cerambycidae). Revista Brasileira de Zoologia, 16: 185-190.

Grazia-Vieira, J., 1972. O gênero Mayrinia Horvath, 1925 (Heteroptera, Pentatomidae, Pentatomini). Revista Peruana de Entomologia, 15: 117-124.

Grimaldi, D. \& M. S. Engel, 2005. Evolution of the insects. New York: Cambridge University Press, 755 p.

Guimarães, J.H., 1961. Terceira contribuição ao conhecimento do gênero Archytas Jaennicke, 1867 (Diptera, Tachinidae). Memórias do Instituto Oswaldo Cruz, 59: 355-395.

Guimarães, J.H., 1963. Quarta contribuição ao conhecimento do gênero Archytas Jaennicke, 1867 (Diptera, Tachinidae). Memórias do Instituto Oswaldo Cruz, 61: 153-164.

Gumovsky, A. \& Z. Boucek, 2003. A new genus of Entedoninae (Hymenoptera: Eulophidae) from Brazil. Neotropical Entomology, 32: 443-447.

Hansson, C., 2000. Description of a new genus of Entedoninae (Hymenoptera: Eulophidae) from the Neotropical Region, including three new species. Journal of Hymenoptera Research, 9: 313-319.

Hermann, L.H., 1979. Revision of Stereocephalus (Coleoptera, Staphylinidae, Paederinae). American Museum Novitates, 2683: 1-20.

Hickel, E.R., J.P.H.J. Ducroquet, R.P. Leite-Jr. \& R.M.V.B.C. Leite, 2001. Fauna de Homoptera: Auchenorrhyncha em pomares de ameixeira em Santa Catarina. Neotropical Entomology, 30: 725-729.

Hovore, F.T. \& A. Santos-Silva, 2007. Variação do colorido elitral, nova espécie e novos registros em Cometes AudinetServille, 1828 (Coleoptera, Cerambycidae, Disteniinae). Papéis Avulsos de Zoologia, 47: 113-125.

Irmler, U., 2003. Distribution redescription and description of new species of the Clavilispinus exiguus group (Coleoptera: Staphylinidae, Osoriinae) in the Neotropical region. Amazoniana, 17: 349-360.

Irmler, U., 2009. A second species for the genus Craspedus Bernhauer, 1908 from the Neotropical region (Coleptera, Staphylinidae, Osoriinae). Dugesiana, 16: 75-78.

Jesús-Bonilla, V.S., J.F. Nunes, A.M. Penteado-Dias \& A. ZaldívarRiverón, 2011. A new synonym of the Neotropical parasitoid wasp genus Notiospathius (Braconidae, Doryctinae), with redescription of two species and description of five new species from Brazil. ZooKeys, 122: 71-90.

Johnson, N.F., 1981. The New World Species of the Telenomus nigricornis group (Hymenoptera: Scelionidae). Annals of the Entomological Society of America, 74: 73-78.

Juñent, S.R. \& M.S. Loiacono, 1995. Los ejemplares tipo de Carabidae y Haliplidae (Adephaga, Coleoptera) depositados em La coleccion del Museo de La Plata. Revista do Museo de La Plata, 21: 1-16.

Kerzhner, I.M. \& F.V. Konstantinov, 2008. Species of the genus Pagasa from Central and South America (Heteroptera, Nabidae). Zoosystematica Rossica, 17: 37-52.

Kimsey, L.S., 1985. Distinction of the Neochrysis (Chrysididae, Hymenoptera). Psyche, 92: 269-286.

Klein, R.M., 1978. Mapa fitogeográfico do Estado de Santa Catarina. Itajaí, Herbário Barbosa Rodrigues, 24p.

Krug, C. \& I. Alves-dos-Santos, 2008. O uso de diferentes métodos para amostragem da fauna de abelhas (Hymenoptera, Apidae), um estudo em Floresta Ombrófila Mista em Santa Catarina. Neotropical Entomology, 37: 265-278.

Lamas, C.J.E. \& G.B. Mellinger, 2008. A new species of Cnodalomyia Hull, 1962 (Diptera, Asilidae, Asilinae) from Brazil. Zootaxa, 1676: 37-43.

Loiácono, M.S., N.B. Diaz, F.E. Gallardo \& C.B. Margaria, 2001. The types of Encyrtidae (Hymenoptera: Chalcidoidea) housed at the Museo La Plata, Argentina. Revista de la Sociedad Entomológica Argentina, 60: 147-161.

Lugo-Ortiz, C.R. \& W.P. McCafferty, 1996a. The genus Paracloeodes (Insecta: Ephemeroptera: Baetidae) and its presence in South America. Annales de Limnologie, 32: 161- 
169.

Lugo-Ortiz, C.R. \& W.P. McCafferty, 1996b. Taxonomy of the Neotropical genus Americabaetis, new status (Insecta: Ephemeroptera: Baetidae). Studies on Neotropical Fauna and Environment, 31: 156-169.

Lutinski, J.A. \& F.R.M. Garcia, 2005. Análise faunística de Formicidae (Hymenoptera: Apocrita) em ecossistema degradado no município de Chapecó, Santa Catarina. Biotemas, 18: 73-86.

Lutinski, J.A., F.R.M. Garcia, C.J. Lutinski \& S. Iop, 2008. Diversidade de formigas na Floresta Nacional de Chapecó, Santa Catarina, Brasil. Ciência Rural, 38: 1810-1816.

Lutinski, C.J., J.A. Lutinski, M.K.M. Costa \& F.R.M. Garcia, 2011. Análise faunística de gafanhotos na Floresta Nacional de Chapecó, Santa Catarina. Pesquisa Florestal Brasileira, 31: 43-50.

Marcondes, C.B., A. Fernandes \& G.A. Muller, 2006. Mosquitoes (Diptera: Culicidae) near a reservoir in the Western part of the Brazilian State of Santa Catarina. Biota Neotropica, 6: 0180.

Marinoni, R.C., L.M. Almeida, D.S. Napp \& G.H. Rosado-Neto, 1992. Primeira lista do material-tipo de Coleoptera da coleção de entomologia de Pe. J.S. Moure, do Departamento de Zoologia da Universidade Federal do Paraná. Revista Brasileira de Zoologia, 9: 99-126.

Martins, U.R., 1971. Notas sobre Cerambycinae (Coleoptera, Cerambycidae) VI. Subsídios ao conhecimento dos Ibiodini. Arquivos de Zoologia, 21: 121-178.

Martins, U.R. \& M.H.M. Galileo, 1997. Revisão dos gêneros Pseudestola Breuning, Estolomimus Breuning e Euestola Breuning (Coleoptera, Cerambycidae, Lamiinae, Demiphorini). Revista Brasileira de Zoologia, 14: 99-112.

Martins, U.R. \& M.H.M. Galileo, 1995. Os gêneros Coeloprocta Aurivillius; Anisopleplus Melzer e Acaua gen.n. (Coleoptera, Cerambycidae, Lamiinae, Desmiphorini). Revista Brasileira de Zoologia, 12: 25-29.

Martins, U.R. \& M.H.M. Galileo, 2008. Notas e novos táxons em Acanthoderini (Coleoptera: Cerambycidae: Lamiinae). Revista Brasileira de Zoologia, 25: 507-511.

Martins, U.R. \& M.H.M. Galileo, 2001. Descrições, transferências e notas em Apomecynini (Coleoptera, Cerambycidae, Lamiinae). Revista Brasileira de Zoologia, 18: 1227-1235.

Martins, U.R. \& M.H.M. Galileo, 1996. Descrições e notas sobre Cerambycidae (Coleoptera) sul-americanos. Revista Brasileira de Zoologia, 13: 291-311.

Meinander, M., 1974. Coniopterygidae from South and Central America (Neuroptera). Notulae Entomologicae, 54: 97-106.

Mello, G.A.R., A.P. Aguiar, B.R. Garcete-Barret, 2012. Hymenoptera. p. 553-612. In: Rafael, J.A., G.A.R. Melo, C.J.B. Carvalho, S.A. Casari \& R. Constantino (Eds.). Insetos do Brasil: Diversidade e Taxonomia. Ribeirão Preto: Holos, 796 .

Milleo, J., L.M. Almeida \& I.M.M. Lima, 1997. Contribuição ao estudo de Brachiacanthadini (Coleoptera, Coccinellidae, Hyperaspinae). Revista Brasileira de Zoologia, 14: 391-405.

Molineri, C., 2001. El gênero Tricorythopsis (Ephemeroptera: Leptohyphidae): nuevas combinaciones y descripción de nuevas especies y estadios. Revista de la Sociedad Entomológica Argentina, 60: 217-238.

Monteiro, R.C. \& L.A. Mound., 2012. Thysanoptera. p. 407-422. In: Rafael, J.A., G.A.R. Melo, C.J.B. Carvalho, S.A. Casari \& R. Constantino (Eds.). Insetos do Brasil: diversidade e taxonomia. Ribeirão Preto, Holos Editora, 81op.

Moreira, F.F.F., J.R.I. Ribeiro, J.L. Nessimian, M.M. Itoyama, M.M.U. Castanhole \& L.L.V. Pereira, 2011. New records and distribution expansions for Neotropical water-striders (Insecta: Heteroptera: Gerromorpha). Check List, 7: 303309.

Musetti, L. \& N.F. Johson, 2004. Revision of the New World species of the genus Monomachus Klug (Hymenoptera: Proctotrupoidae, Monomachidae). The Canadian
Entomologist, 136: 501-552.

Napp, D.M., 2009. Revisão das espécies sul-americanas de Rhopalophora (Coleoptera, Cerambycidae). Zoologia, 26: 343-356.

Napp, D.M., 2007. Revisão do gênero Aglaoschema Napp (Coleoptera, Cerambycidae). Revista Brasileira de Zoologia, 24: 793-816.

Napp, D.M. \& D.T. Reynaud, 1998. New species of Chariergus White and Ethemon Thornson (Coleoptera, Cerambycidae, Compsocerini). Insecta Mundi, 12: 155-159.

Nieser, N. \& M. Lopez-Ruf, 2001. A review of Limnocoris Stal (Heteroptera: Naucoridae) in Southern South America East of the Andes. Tijdschrift voor Entomologie, 144: 261-328.

Nieser, N. \& P. Chen, 2002. Six new species of Neotrephes China, 1936 (Heteroptera: Helotrephidae) from Brazil, with a key to Neotropical Helotrephidae. Lundiana, 3: 31-40.

Nihei, S.S. \& M.P. Pansonato, 2006. Revision of Prophorostoma Townsend, 1927 (Diptera, Tachinidae, Dexiinae), with the description of a new species. Papéis Avulsos de Zoologia, 46: 239-244.

Nunez, E. \& M.S. Couri, 2002. Redescrição de sete espécies de Chrysotachina Brauer \& Bergenstamm (Diptera, Tachinidae) para a América do Sul. Revista Brasileira de Zoologia, 19: 1-18.

Penteado-Dias, A.M., 1996. New Neotropical species of the genus Meteoridea Ashmead (Hymenoptera: Braconidae: Meteorideinae). Zoologische Mededelingen, 70: 197-205.

Rafael, J.A., G.A.R. Melo, C.J.B. Carvalho, S.A. Casari, R. Constantino, 2012. Insetos do Brasil: Diversidade $\mathrm{e}$ Taxonomia. Ribeirão Preto: Holos, 796 pp.

Ravanello, C.T., 2007. Diversidade e abundância de larvas de Odonata (Insecta) em nove rios da Bacia Hidrográfica do Alto Rio Uruguai - Santa Catarina. Dissertação (Mestrado em Ciências Ambientais). Universidade Comunitária Regional de Chapecó. 56p.

Reichardt, H., 1964. On Neotropical Carabidae (Coloeptera). Psyche, 71: 49-52.

Ribeiro, J.R.I. \& A.L. Estévez, 2009. The small species of Belostoma Latreille (Heteroptea, Belostomatidae). III. A revision of oxyurum group, with a new species from Brazil and description of the male of $B$. noualhieri Montandon. Revista Brasileira de Entomologia, 53: 207-215.

Rotheray, G.E., M. Zumbado, E.G. Hancock \& F.C. Thompson, 2000. Remarkable aquatic predators in the genus Ocyptamus (Diptera, Syrphidae). Studia Dipterologica, 7: 385-389.

Santos-Silva, A. \& U.R. Martins, 2004. Notas e descrições em Disteniidae (Coleoptera, Cerambycidae). Revista Brasileira de Zoologia, 21: 145-152.

Shaffer,J.C., 1991. Redescription and reassignment of the brazilian Anerastia hemirhodella Hampson to Volatica Heinrich (Pyralidae: Phycitinae). Journal of the Lepidorologists' Society, 45: 124-129.

Shimbori, E.M. \& A.M. Penteado-Dias, 2011. Taxonomic contribution to the Aleiodes melanopterus species-group (Hymenoptera, Braconidae, Rogadinae) form Brazil.ZooKeys, 125: 15-25.

Shpeley, D. \& G.E. Ball, 2008. Taxonomic review of Neotropical Tetragonoderus quadriguttatus assemblage (Coleoptera, Carabidae, Cyclosomini) with descrition of $T$. deuvei, new species, and New Indian and Neartic locality records. Insecta Mundi, 50: 1-16.

Silva, B.R., 1907. Lepidópteros do Brasil. Rio de Janeiro, Imprensa Nacional. 330p.

Silva, R.R., 1999. Formigas (Hymenoptera, Formicidae) do oeste de Santa Catarina: histórico das coletas e lista atualizada das espécies do Estado de Santa Catarina. Biotemas, 12: 75-100.

Silva, R.R. \& R. Silvestre, 2004. Riqueza da fauna de formigas (Hymenoptera: Formicidae) que habita as camadas superficiais do solo em Seara, Santa Catarina. Papéis Avulsos de Zoologia, 44: 1-11.

Silva, R.R. \& R. Silvestre, 2000. Diversidade de formigas 
(Hymenoptera: Formicidae) em Seara, oeste de Santa Catarina. Biotemas, 13: 85-105.

Scarbrough, A.G., 2008. New Ommatius Wiedemann from the Americas with two new species group, keys, and taxonomic notes (Diptera, Asilidae). Insecta Mundi, 32: 1-14.

Stebnicka, Z., 2003. The new world species of Ataenius Harold, 1867. III. Revision of the A. imbricatus - group sensu lato (Coleoptera, Scarabaeidae, Aphodiinae, Eupariini). Acta Zoologia Cracoviensia, 46: 219-249.

Stebnicka, Z., 2004. The new world species of Ataenius Harold, 1867. IV. Revision of the $A$. strigicauda-group (Coleoptera, Scarabaeidae, Aphodiinae, Eupariini). Acta Zoologia Cracoviensia, 47: 211-228.

Stebnicka, Z., 2005. The new world species of Ataenius Harold, 1867. IV. Revision of the A. aequalis-platensis-group (Coleoptera, Scarabaeidae, Aphodiinae, Eupariini). Acta Zoologia Cracoviensia, 48B: 99-138.

Stebnicka, Z., 2007. The new world species of Ataenius Harold, 1867. VIII. Revision of the $A$. scutellaris-group and diagnosis of the A. texanus-carinator group with descriptions of new species (Coleoptera, Scarabaeidae, Aphodiinae, Eupariini). Acta Zoologia Cracoviensia, 50B: 45-81.

Toma, R., 2001. Descrição de uma espécie nova de Thysanopsis (Diptera, Tachinidae). Iheringia, Série Zoologia, 91: 37-40.

Toma, R. \& J.H. Guimarães, 2001. Moreiria wiedemanni sp. nov. e redescrição de $M$. maura (Diptera, Tachinidae). Iheringia, Série Zoologia, 91: 49-52.

Toma, R., 2003. Estudo das espécies do "complexo Acaulona" sensu Sabrosky (Diptera, Tachinidae). Revista Brasileira de Entomologia, 47: 267-282.

Trematerra, P. \& J.W. Brown, 2004. Argentine Argyrotaenia (Lepidoptera: Tortricidae): synopsis and descriptions of two new species. Zootaxa, 574: 1-12.

Triplehorn, C.A. \& N.F. Johnson, 2011. Estudo dos Insetos. São Paulo, Cengage Learning, 8o8p.

Uehara-Prado, M., A.V.L. Freitas, R.B. Francini \& K.S. Brown-Jr, 2004. Guia das borboletas frugívoras da Reserva Estadual do Morro Grande e região de Caucaia do Alto, Cotia (São Paulo). Biota Neotropica, 4: 1-25.

Urban, D., 2002. Espécies novas de Anthodioctes Holmberg e notas sobrealguns Dianthidiini(Hymenoptera,Megachilidae). Revista Brasileira de Zoologia, 19: 151-158.

Urban, D., 1993. Gnathanthidium, gen.n. de Anthidiinae na América do Sul (Hymenoptera, Megachilidae). Revista Brasileira de Zoologia, 9: 337-343.
Urban, D., 1995a. Moureanthidium, gen.n. de Dianthidiini no Brasil (Hymenoptera, Megachilidae). Revista Brasileira de Zoologia, 12: 37-45.

Urban, D., 1995b. Espécies novas de Lanthanomelissa Holmberg e Lanthanella Michener \& Moure (Hymenoptera, Anthophoridae, Exomalopsinae). Revista Brasileira de Zoologia, 12: 767-777.

Ururahy-Rodrigues, A., 2004. Artemita bicolor Kertész, novo sinônimo de Artemita podexargenteus Enderlein, (Diptera, Stratiomyidae) com notas nas terminálias masculina e feminina. Revista Brasileira de Zoologia, 21: 397-402.

Wendt, L.D. \& C.J.B. Carvalho, 2007. Taxonomia de Fanniidae (Diptera) do sul do Brasil - I: nova espécie e chave de identificação de Euryomma Stein. Revista Brasileira de Entomologia, 51: 197-204.

Wendt, L.D. \& C.J.B. Carvalho, 2009. Taxonomia de Fanniidae (Diptera) do sul do Brasil - II: novas espécies e chave de identificação de Fannia Robineau-Desvoidy. Revista Brasileira de Entomologia, 53: 171-206.

Wirth, W.W., 1991. Forcipomyia bicolor and related species of the subgenus Lepidohelea in Brazil (Diptera, Ceratopogonidae). The Florida Entomologist, 74: 506-517.

Wirth, W.W. \& G.R. Spinelli, 1992. The american species of the annulatipes group of the subgenus Lepidohelea, genus Forcipomyia (Diptera, Ceratopogonidae). Insecta Mundi, 6: 109-125.

Wirth, W.W. \& M.L. Felippe-Bauer, 1989. The neotropical biting midges related to Culicoides paraensis (Diptera, Ceratopogonidae). Memórias do Instituto Oswaldo Cruz, 84: 551-565.

Zaballos, J.P. \& J. Mateus, 1997. Dos nuevos Anillinus Casey, 1918 (Coleoptera, Caraboidea, Trechidae) de Brasil. Elytron, 11: $133-143$.

Zanol, K.M.R., 2003. Frequenamia (Cicadellidae, Deltocephalinae): distribuição geográfica, notas sinonímicas e descrições de dezessete novas espécies. Acta Biológica Paranaense, 32: 9-67.

Zikan, W. \& P. Wygodzinsky, 1948. Catálogo dos tipos de insetos do Instituto de Ecologia e Experimentação Agrícolas. Boletim do Serviço Nacional de Pesquisas Agronômicas, 4: 1-97.

\section{Recebido em: 26/o8/2012}

Aceito em: 04/01/2013

\section{Como citar este artigo:}

Favretto, M.A., E.B. Santos \& C.J. Geuster, 2013. Entomofauna do Oeste do Estado de Santa Catarina, Sul do Brasil. EntomoBrasilis, 6(1): 42-63. Acessível em: http://www.periodico.ebras.bio.br/ojs/index.php/ebras/article/view/271. doi:10.12741/ebrasilis.v6i1.271
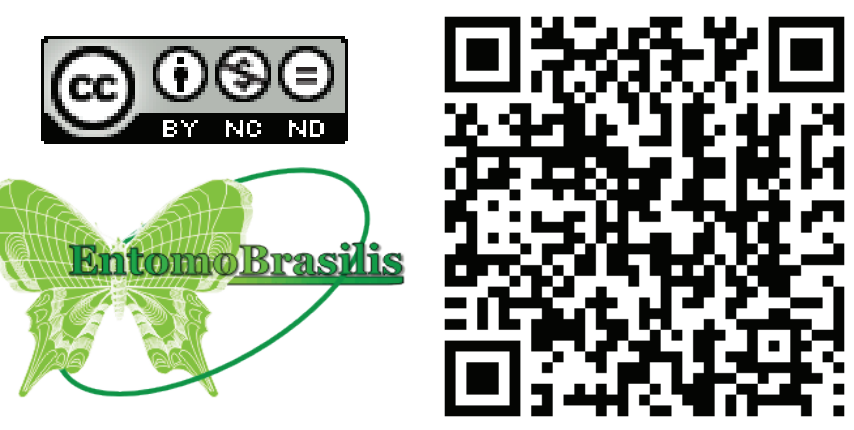